\title{
ESTRATIGRAFÍA Y PALEONTOLOGÍA DEL YACIMIENTO QUIN-18 (FORMACIÓN VALMAYOR, FRASNIENSE, SINCLINAL DE ALMADÉN, ESPAÑA)
}

\author{
Rodolfo GOZALO ', Miguel V. PARDO ALONSO ${ }^{2}$ \\ y José Ignacio VALENZUELA RÍOS ${ }^{3}$
}

\author{
Departamento de Geología. Universitat de València. C/ Dr. Moliner 50. E-46100-Burjassot \\ (Valencia) ESPAÑA. \\ 'Rodolfo.Gozalo@uv.es =Miguel.V.Pardo@uv.es.Jose.I.Valenzuela@uv.es
}

Gozalo, R., Pardo Alonso, M.V. y Valenzuela Ríos, J.I. 2002. Estratigrafía y paleontología del yacimiento QUIN-18 (Formación Valmayor, Frasniense, Sinclinal de Almadén, España). [Stratigraphy and palaeontology of the QUIN-18 site (Valmayor Formation, Frasnian, Almadén Syncline, Spain).] Revista Española de Paleontología, 17(1), 83-99. ISSN 0213-6937.

\begin{abstract}
A thick volcaniclastic succession (Complejo Vulcano-Sedimentario de Chillón) that overlies a mainly pelitic succession (Valmayor Fm.), both of Frasnian age, crops out in the core of the Almadén Syncline (Ciudad Real, Spain). QUIN-18 outcrop is located at the top of the Valmayor Fm, and has yielded abundant ostracods and brachiopods indicating an age comprised between Middle and Upper Mesotaxis asymmetricus conodont zones (upper part of Palmatolepis punctata to Early P. hassi in the standard conodont zones respectively).

Ostracods belong to the so-called Eifel Ökotyp indicating a peri-reefal, sublittoral, marine platform biotope. Brachiopods Pradochonetes muelleri and Douvillina alvarezi, which are considered as autochthonous or parautochthonous, indicate general marine sub-littoral conditions also, but with lower energy and soft sea-floor, below common storm base wave. The other brachiopod elements lead to believe that they were allochthonous elements transported to QUIN-18 by occasional storms stronger than usual. They, together with ostracods, would have lived in shallower environments, probably near small reefal patches on the slopes of volcanoes submarine hills that were represented by the Complejo Vulcano-Sedimentario.

In brief, upper levels of Valmayor Fm. would be deposited in a low energy sheltered sublittoral environment, below common storm wave base on a very little consolidated ocean floor and high sedimentation rate; these are the limiting conditions that would allow the development of a hardly diversified autochthonous fauna; however, storms could occasionally bring allochthonous faunas from nearby shallower areas.
\end{abstract}

Keywords: Ostracoda, Brachiopoda, biostratigraphy, taphonomy, palaeoecology, Frasnian, CentralIberian Zone, Spain.

\section{RESUMEN}

En el núcleo del Sinclinal de Almadén (Ciudad Real, España) aflora una potente sucesión vulcano-sedimentaria (Complejo Vulcano-Sedimentario de Chillón), que se apoya sobre una sucesión esencialmente limo-pelítica (Fm. Valmayor), ambas frasnienses, en el techo de esta última se encontró el yacimiento QUIN-18, especialmente rico en braquiópodos y ostrácodos fósiles. La edad indicada por éstos correspondería a la parte alta de la Zona de Mesotaxis asymmetricus Media hasta la Zona de M. asymmetricus Superior (parte alta de la Zona de Palmatolepis punctata a la Zona de P. hassi Inferior, en la zonación estándar de conodontos).

Los ostrácodos pertenecen al llamado ecotipo (ökotyp) eifélico, e indican un biotopo peri-arrecifal en una plataforma marina infralitoral. Sin embargo, los braquiópodos dominantes, Pradochonetes muelleri y Douvillina alvarezi, considerados autóctonos o parautóctonos, indicarían un ambiente infralitoral protegido, de escasa energía y fondos blandos, por debajo del nivel de base habitual de las olas de tormenta. El resto de braquiópodos, considerados alóctonos, serían transportados al yacimiento en el curso de fuertes tormentas ocasionales; éstos, junto con los ostrácodos, habrían vivido en medios más someros, probablemente cerca de pequeños parches arrecifales en laderas de volcanes submarinos (Complejo Vulcano-Sedimentario). 
En conclusión, la Fm. Valmayor representa aquí un ambiente marino sublitoral protegido, de escasa energía, y por debajo del nivel de base de olas de tormenta normales, con un substrato poco consolidado y tasas de sedimentación medias o altas, donde se desarrollaría una fauna autóctona poco diversificada. Ocasionalmente, tormentas excepcionales harían llegar formas alóctonas, arrastradas desde áreas próximas más someras.

Palabras clave: Ostracoda, Brachiopoda, bioestratigrafía, tafonomía, paleoecología, Frasniense, Zona Centroibérica, España.

\section{ABRIDGED ENGLISH VERSION}

A thick volcaniclastic succession (Complejo VulcanoSedimentario de Chillón) that overlies a mainly pelitic succession (Valmayor Fm.), both of Frasnian age, crops out in the core of the Almadén Syncline (Ciudad Real, Spain). In its eastern periclinal end, the volcaniclastic Complex laterally changes to pelitic rocks of the Valmayor Fm., which are the youngest rocks of this unit found in the Almadén Syncline (Pardo Alonso, 1997).

In the core of this syncline, pelitic horizons of Valmayor Fm. (Pardo Alonso y García-Alcalde, 1996) frequently contain brachiopods, which are mainly tectonically deformed. However, an outstanding outcrop (QUIN-18) has been found in its eastern periclinal end. This outcrop has yielded a rich, well preserved and not much deformed fossil fauna of brachiopods and ostracods with very well preserved shells.

This study encompasses systematics, biostratigraphic, taphonomic, palaeoecologic and palaeogeographic aspects of the fossil and lithological record exposed at QUIN-18.

Brachiopod association consists of internal and external casts of complete shells and isolated valves of the following taxa: Pradochonetes muelleri, Douvillina alvarezi, Ripidiorhynchus aff. kotalensis, Cariniferella cf. dumontiana and Productella subaculeata. It is not rear to find articulated exemplars of the two former species that also show variety of sizes.

Ostracods belong to Hollinella (Keslingella)? sp., Polyzygia neodevonica neodevonica, Svantovites inops, Bythocyproidea weyanti, Ponderodyctia cf. belliloci and some kirkbyids and podocopids that because of bad preservation could not be identified at a lower rank. Comparable associations have been found in Ardennes and Boulonnais (Becker, 1971; Lethiers, 1982, 1984; Casier, 1987), in the Cantabrian Mountains (Becker, 1988, 1995) and in the Iberian Chains (Gozalo, 1994).

Svantovites inops and Bythocyproidea weyanti are clearly Frasnian; and, more precisely, $S$. inops indicates DSO2 Zone that was established by Lethiers (1984) in the Ardennes and Boulonnais. This zone was correlated with part of the lower Mesotaxis (former Polygnathus) asymmetricus conodont Zone and with the Middle and Upper M. asymmetricus zones; they correspond to Palmatolepis transitans, $P$ punctata and Early P. hassi standard conodont zones respectively. The presence of Pradochonetes muelleri allows correlation of these faunistic levels with the $P$. muelleri Range Biozone of Pardo and García Alcalde (1984); this zone is Frasnian and was tentatively correlated with the upper part of
Middle M. asymmetricus and Upper M. asymmetricus zones (Pardo and García Alcalde, op. cit.). Thus, ages indicated by ostracods and brachiopods are congruent.

Ostracods belong to the so-called Eifel Ökotyp indicating a peri-reefal, sublittoral, marine platform biotope and could be allochthonous elements transported to QUIN-18 from shallower settings. Brachiopods indicate general marine sub-littoral conditions, as well. The good preservation and large size variability of $P$. muelleri and D. alvarezi, evoke an authochthonous character of these forms and suggest that the environment where the outcrop was formed was quiet, probably in a protected setting. On the other hand, the fragmentation and biased sizes of other brachiopods elements lead to believe that they were allochthonous elements as well as the ostracods that were brought to the basin from shallower settings by occasional storms stronger than usual.

According to the palaeoecologic interpretations presented above and the lithology of QUIN-18, the youngest levels of the Valmayor Fm. could be deposited in a marine, sublittoral, sheltered, quiescent environment, below the normal storm wave base. Medium to high sedimentary rates on unconsolidated sediment with few or no bioturbation at all characterized this setting. In these conditions the life-style of the autochthonous douvilliniids would be interpreted as resting freely on the soft ground on its ventral valves. The QUIN-18 environment seems very close to that of the taphofacies VII defined by Speyer and Brett (1991) in the Devonian Hamilton Group of New York. The main difference would be the lack of pelagic faunas in Spain. This fact could possibly be interpreted as an indication of restricted communication possibilities with the open sea by the occurrence of a barrier. This barrier could be represented for the submarine volcanic cones developed along the platform in the Spanish region. The lateral intertonguing of the Valmayor Fm. and the Complejo VulcanoSedimentario de Chillón supports this interpretation, as well. Some calcareous reefoid patches that occur in the Almadén syncline could be the source area of the allochthonous elements.

\section{INTRODUCCIÓN}

El Sinclinal de Almadén se sitúa en la franja meridional de la Zona Centroibérica (Fig. l A y lB), concretamente en su parte media. En el núcleo afloran, junto a otros más antiguos, materiales del Devónico que llegan hasta la Zona de conodontos de Palmatolepis 


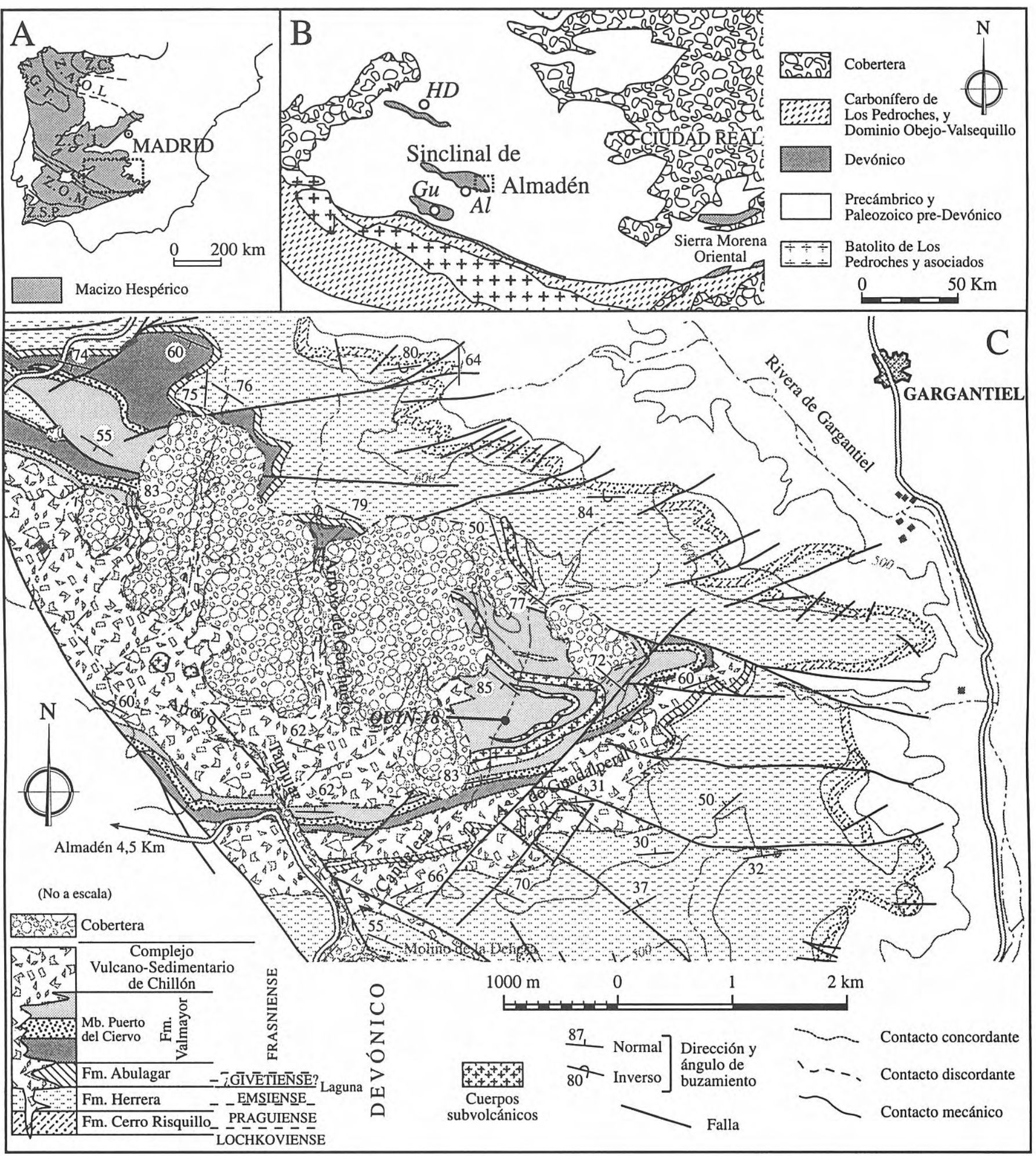

Figura 1.Localización geográfica y geológica del área estudiada. A.- Macizo Hespérico y localización del área ampliada en B, en la rama meridional de la Zona Centroibérica. B.- Localización del Sinclinal de Almadén, con indicación de su terminación oriental (ampliada en C). C.- Mapa geológico detallado del entorno del yacimiento QUIN-18, en la terminación oriental del Sinclinal de Almadén (según Pardo Alonso, 1997).

Geographical and geological setting. A.- Hesperian Massif with location of the studied area, enlarged in B, within the southern Central-Iberian Zone. B.- Syncline of Almadén with its eastern pericline end (amplified in C). C.Detailed geological map of the surroundings of QUIN-18 outcrop in the eastern end of Almadén Syncline (after Pardo Alonso, 1997). 
linguiformis del Frasniense terminal (Pardo y GarcíaAlcalde, 1984; García-López et al., 1999). La serie devónica es mayoritariamente detrítica en su parte inferior, mientras que la superior consiste en un complejo volcánico efusivo, denominado Complejo VulcanoSedimentario (CVS) de Chillón (Pardo Alonso, 1997); los materiales volcánicos son mayoritarios en esta parte de la serie, pero se encuentran también intercalados con frecuencia variable entre los materiales detríticos del resto del Devónico. El CVS corresponde a edificios volcánicos, en su mayor parte submarinos, que debieron tener su foco a la altura de las actuales poblaciones de Chillón y Almadén (Vergés, 1983).

La mayoría de los estudios estratigráficos y paleontológicos sobre estas series se han centrado en la parte media del sinclinal y parcialmente en la terminación occidental (clásicamente conocida como "Sinclinal de Garlitos"). Sin embargo, la serie devónica de la terminación oriental, hasta el momento no había sido objeto de estudio, salvo de un modo muy reducido (por ejemplo, las calizas emsienses del Molino de la Dehesa; Pardo y García-Alcalde, 1984).

En la terminación oriental del sinclinal, los niveles volcánicos del Frasniense son más escasos que en la parte media del mismo y la serie volcánica pasa lateralmente a niveles pelíticos, con esporádicas intercalaciones de areniscas y limolitas, que corresponden localmente a los niveles más altos de la Fm. Valmayor (Pardo Alonso y García-Alcalde, 1996; Pardo Alonso, 1997). En estos niveles se ha localizado un yacimiento excepcional, el QUIN-18, con una excelente conservación del material fósil, principalmente de los ostrácodos y braquiópodos, en general en forma de moldes internos y externos, dentro de pelitas con escasa deformación tectónica; la especial asociación de elementos faunísticos y el hecho de situarse en niveles detríticos que son equivalentes laterales de las series vulcano-sedimentarias del resto del sinclinal, lo hacen sumamente interesante. Hay que destacar que la fauna de ostrácodos encontrada es similar a la de otros niveles givetienses y frasnienses de las Ardenas y Boulonnais, en Francia (Becker, 1971; Lethiers, 1982, 1984; Casier, 1987), Cordillera Cantábrica (Becker, 1988, 1995) y Cadenas Ibéricas (Gozalo, 1994), mientras que los braquiópodos mayoritariamente pertenecen a formas definidas en la región.

Este estudio recoge observaciones preliminares sobre la paleoecología y tafonomía del yacimiento, la discusión de varias de las formas más características, especialmente de algunos de los ostrácodos, y la reconstrucción de conjunto de los ambientes asociados a los edificios volcánicos submarinos que se desarrollaron en la región durante la parte alta del Frasniense.

\section{ESTRATIGRAFÍA DEL FRASNIENSE EN EL SINCLINAL DE ALMADÉN}

El Frasniense se inicia en la región de Almadén (Fig. 2A) con una serie cuarcítica, la Fm. Abulagar, a la que sigue una espesa unidad de predominancia pelítica, Fm. Valmayor
(Pardo Alonso y García-Alcalde, 1996; Pardo Alonso 1997), que contiene una importante y continua intercalación arenoso-cuarcítica (Mb. Puerto del Ciervo; Pardo Alonso, op. cit.); en la mayor parte del Sinclinal de Almadén, el miembro superior de esta formación es sucedido por una potente unidad de carácter volcánico, el CVS de Chillón antes mencionado; éste, compuesto principalmente por piroclastos y algunas coladas de lavas, ha sido interpretado como una superposición de conos volcánicos submarinos (Vergés, 1983). La unidad volcánica contiene en su parte media-alta esporádicas intercalaciones de niveles calcáreos de escasa continuidad, algunos de los cuales están formados principalmente por corales coloniales; cabe destacar entre estos últimos el yacimiento V-960, citado por Pardo y García-Alcalde (1984: p. 86).

En la terminación oriental del sinclinal de Almadén, la sucesión es algo diferente en lo que respecta a las relaciones entre el CVS de Chillón y la Fm. Valmayor. Allí, en la serie del arroyo de la Candelera (Figs. 1C y 2B), tras un episodio volcánico inicial, retorna la sedimentación pelítica típica del miembro superior de la Fm. Valmayor; la unidad volcánica aflora en las proximidades, a unos $400 \mathrm{~m}$ al oeste (Fig. 1C), pudiéndose observar el paso lateral de una a otra unidad.

A partir de varias columnas estratigráficas y de una cartografía detallada, se ha realizado una sección a lo largo del flanco sur del sinclinal (Fig. 3 A), donde se pueden observar las morfologías y relaciones espaciales entre estas unidades devónicas. Esta reconstrucción, ampliamente basada en una anterior de Vergés (1983), muestra la morfología de los conos volcánicos que constituyen el CVS de Chillón, cuyos focos se situarían aproximadamente entre las localidades actuales de Chillón y Almadén. La acumulación de estos materiales alteraría la morfología del fondo, aproximándolo a la superficie del mar, pudiendo incluso emerger, originando una barrera que delimitaría áreas protegidas en la plataforma. Las zonas alejadas de estos focos (particularmente aquella donde se localiza el yacimiento QUIN-18) se situarían, en principio, a profundidad mayor, sin influencias del vulcanismo. Los niveles calcáreos, con corales, estarían situados en las laderas de los volcanes, sobre fondos más someros que sus equivalentes en edad de la Fm. Valmayor.

Los volcanes se desarrollaron sobre el fondo de un mar epicontinental, relativamente cerca de la costa; ésta, de acuerdo con Vergés (1983) y Pardo Alonso (1997, 1999, 2000), se situaría hacia el ESE, en el área de Sierra Morena Oriental (Fig. 1), de manera que los edificios volcánicos pudieron actuar de barrera, aislando el área donde se depositaron los sedimentos de QUIN-18.

\section{SITUACIÓN DEL YACIMIENTO QUIN-18}

El yacimiento QUIN-18 se sitúa (Fig. 1C) en el Arroyo de la Candelera, a unos $4,5 \mathrm{~km}$ de la población de Gargantiel; sus coordenadas UTM (aproximación de $25 \mathrm{~m}$ ) son:

Hoja MTN E=1:50.000 No 808 (16-32) Almadén. $\mathrm{x}: 508.175$ y: 465.425 .

Estratigráficamente se localiza dentro del miembro superior de la Fm. Valmayor, a unos $320 \mathrm{~m}$ de su base. No se trata de un nivel único sino de varios lechos fosilíferos 


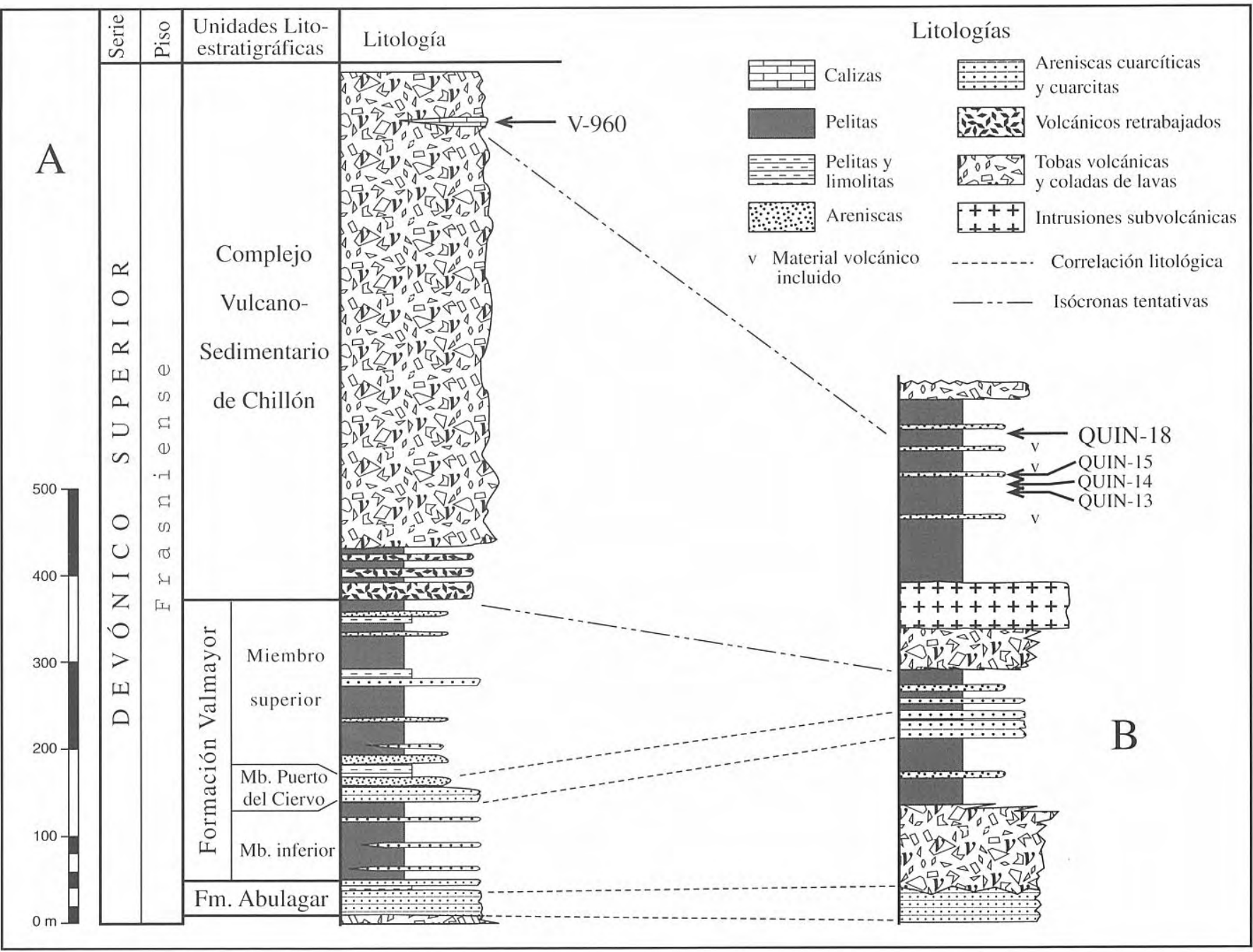

Figura 2. Columnas estratigráficas del Frasniense en el área objeto de estudio: A.- Realizada a la altura de Almadén, en la parte central del Sinclinal de Almadén. B.-Levantada en el $\mathrm{A}^{\mathrm{o}}$ de la Candelera, en la terminación oriental del Sinclinal de Almadén. En A, los niveles del Complejo vulcano-sedimentario de Chillón ocupan más de la mitad de la serie estratigráfica, mientras que en B sus equivalentes en edad son niveles detríticos de la Fm. Valmayor. Los niveles de QUIN-18 son grosso modo correlacionables en edad con los niveles de carbonatos biostromales del yacimiento V-960 (ver Fig. 3).

Frasnian stratigraphic columns: A.- near Almadén in the central part of the Almadén Syncline. B.- Candelera Creek, in the eastern end of the Almadén Syncline. In A beds of the Complejo Vulcano-Sedimentario de Chillón counts for more than half of the stratigraphic sequence, whereas in B their age-equivalent are represented by siliciclastic rocks of the Valmayor Fm. QUIN-18 horizons are grosso modo age-correlated with biostromal carbonates of V-960 (see Fig. 3 ).

situados en pelitas, abarcando un espesor total de unos 50-60 centímetros. En ellos, aparecen braquiópodos, como Pradochonetes muelleri Pardo y García-Alcalde, 1984, Douvillina alvarezi (Pardo y García-Alcalde, 1984), Cariniferella cf. dumontiana (Verneuil, 1850), Ripidiorhynchus aff. kotalensis Brice, 1970 y Productella subaculeata (Murchison, 1840); ostrácodos, como Hollinella (Keslingella) ? sp., Polyzygia neodevonica neodevonica (Matern, 1929), Svantovites inops Becker, 1971, Bythocyproidea weyanti (Becker, 1971), Ponderodictya cf. belliloci Casier, 1986, así como formas indeterminadas de kirkbyidos y podocópidos; trilobites asteropigínidos, crinoideos, bivalvos y braquiópodos inarticulados.

La litología es principalmente argilítica, con pequeñas micas en la superficie de las capas; los colores son grises más o menos obscuros, aunque no hay bandeado de color claramente desarrollado. En general, es raro encontrar restos fósiles en la terminación oriental del sinclinal de Almadén, dentro estas pelitas, por ello destaca el carácter excepcional del yacimiento QUIN-18. De hecho, en todo el miembro superior de la Fm. Valmayor, en el área del arroyo de La Candelera, sólo aparecieron otros tres niveles fosilíferos, mucho más pobres, por debajo de QUIN-18 (Fig. 2B).

Las pelitas grises están finamente laminadas y, además de las micas, presentan en superficie pequeñas partículas de materia orgánica más obscura; salvo dos pequeñas galerías muy cortas, de pocos milímetros de diámetro, no hay señales de bioturbación. Internamente se observan, a veces, láminas milimétricas de material más limolítico e incluso de arenisca, con abundantes bioclastos pequeños. 


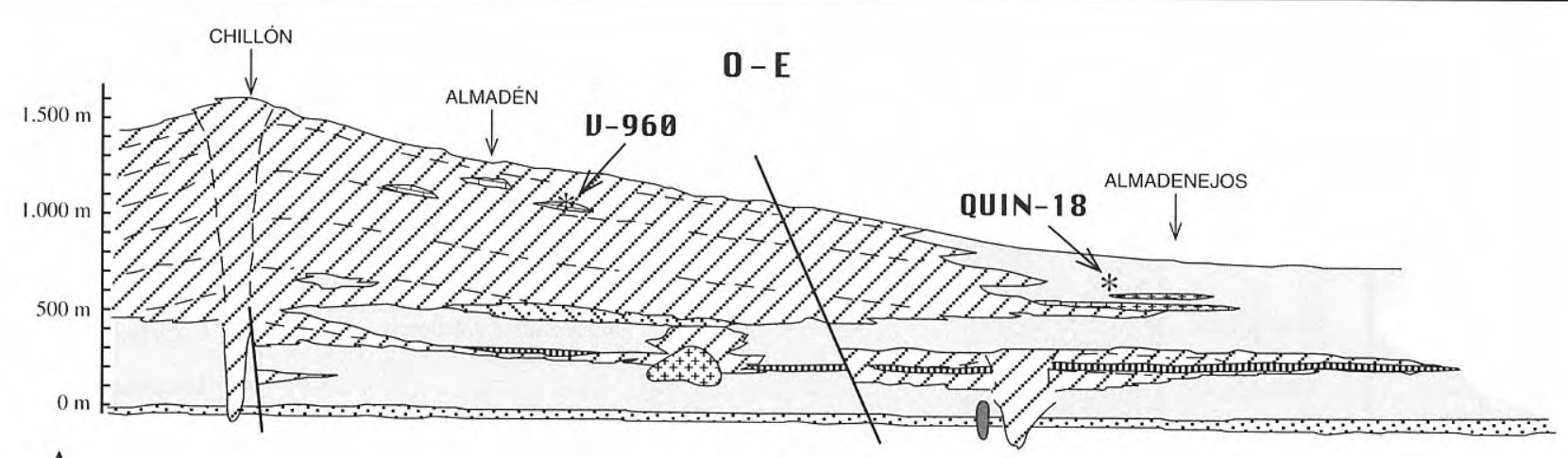

A

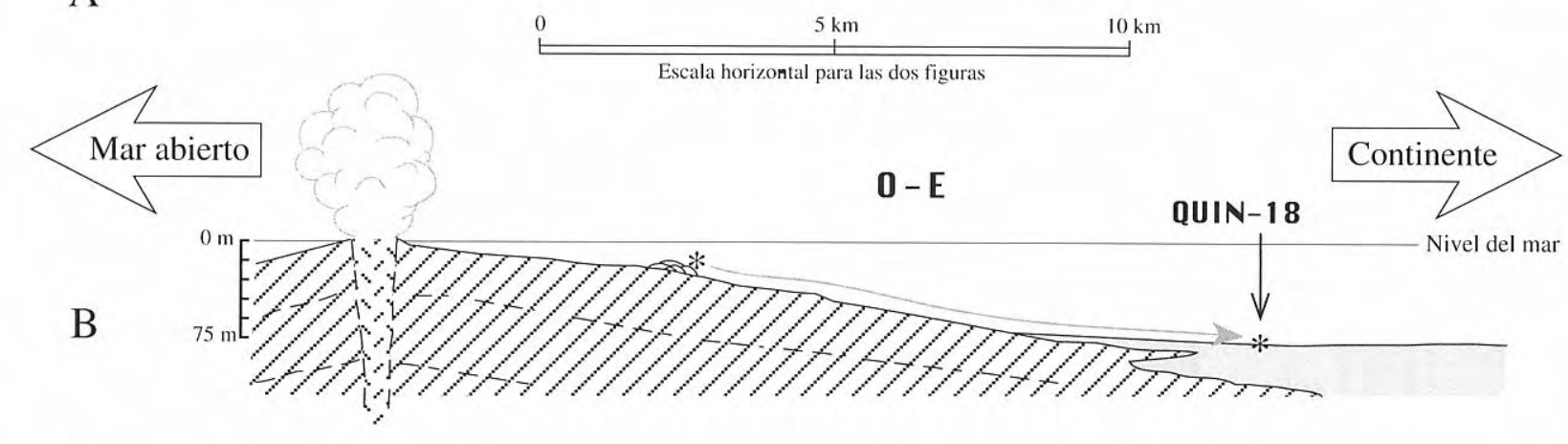

Subvolcánicos

$[\because]$ Chimenea activa

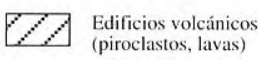

¿ volcánicos retrabajados

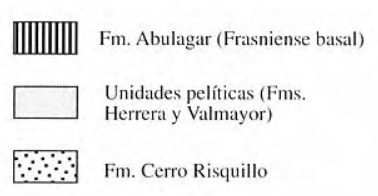

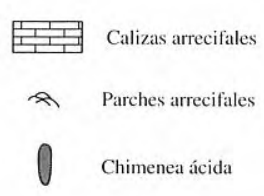

Trayectoria hipotética de los ostrácodos y otros elementos Trayectoria hipotética de los ostrácodos y otros elen
alóctonos, arrastrados por corrientes (itormentas?)

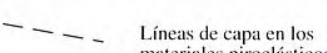

Líneas de capa en los

Figura 3. A.- Reconstrucción de una sección O-E a lo largo del Devónico del flanco sur del Sinclinal de Almadén, modificada de Vergés (1983), con datos cartográficos propios; a la izquierda, los grandes edificios volcánicos, representados por el Complejo vulcano-sedimentario de Chillón; se han representado los niveles de calizas intercalados, algunos de ellos arrecifales (V-960). Los volcánicos pierden espesor hacia el Este, pasando lateralmente a niveles de la Fm. Valmayor, donde se encuentra el yacimiento QUIN-18. B.- Reconstrucción de ambientes a partir del esquema de A (profundidades aproximadas). En las laderas de los volcanes en activo se desarrollarían pequeños parches arrecifales, que serían la fuente de los ostrácodos y otros elementos que luego se encuentran como alóctonos en los niveles de QUIN-18; éstos están situados en una zona posiblemente más profunda y protegida del mar abierto por la barrera de los volcanes.

A.- Reconstruction of a Devonian W-E section along the southern flank of Almadén Syncline, modified from Vergés (1983) with our own cartographic data; left, large volcanic cones represented by the Complejo VulcanoSedimentario de Chillón; interbedded limestone levels, including some reefal ones (V-960), have been represented. Volcanic materials wedge out eastwards, where they laterally change to the Valmayor Fm., which contains QUIN-18 outcrop. B.- Environmental reconstruction from A (depths estimated). On the slopes of the active volcanoes small reefal patches could develop; they would have been the source of ostracods and other elements that are found as allochthonous in QUIN-18, which were located at a protected area, probably deeper and sheltered from the open sea by the volcanoes barrier.

Esporádicamente aparecen también intercalaciones centimétricas de limolitas y areniscas de grano muy fino, con algo de material volcánico, con aspecto de tempestitas distales.

\section{EDAD DEL YACIMIENTO QUIN-18}

De acuerdo con la presencia de $P$. muelleri, estos niveles se encuentran dentro de la biozona de dicha especie (Fig. 4), de edad Frasniense y que fue definida por Pardo y GarcíaAlcalde (1984). En términos de la escala bioestratigráfica de conodontos, dicha Biozona equivale al lapso estratigráfico que incluye la parte alta de la Zona de Mesotaxis asymmetricus (antes Polygnathus asymmetricus) Media y la Zona de M. asymmetricus Superior (o lo que es lo mismo, la parte alta de la Zona de Palmatolepis punctata y la Zona de $P$. hassi Inferior, de la biozonación estándar de conodontos). 


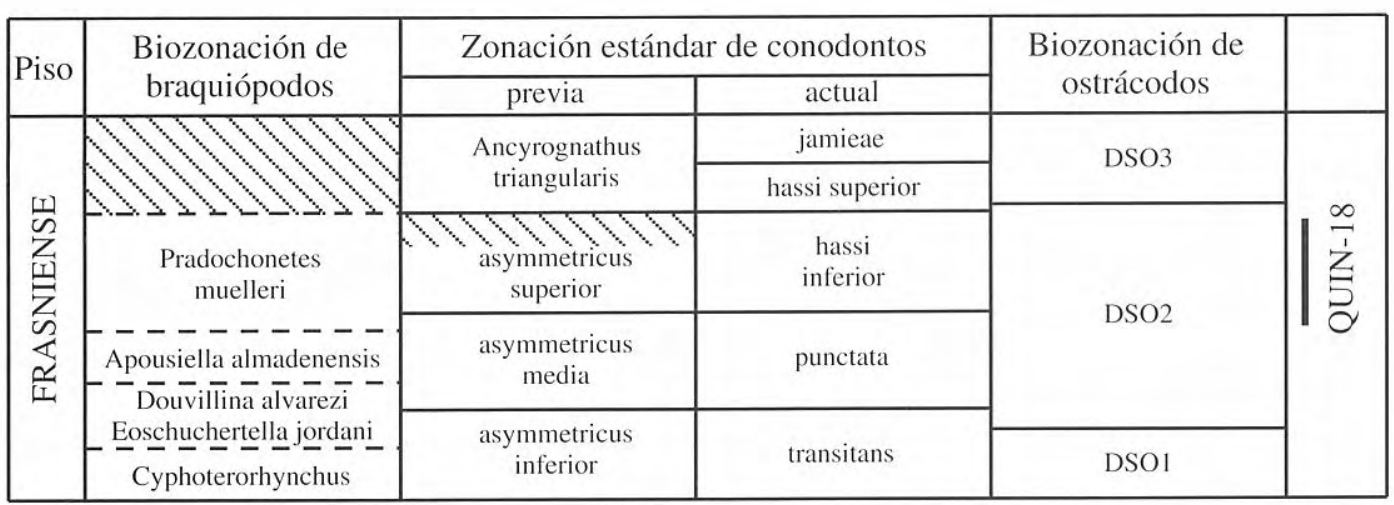

Figura 4. Cuadro de correspondencia para el Frasniense Inferior entre la biozonación de braquiópodos de la región de Almadén (Pardo y García-Alcalde, 1984), las zonaciones previa y actual de conodontos (Ziegler, 1996), la biozonación de ostrácodos propuesta para las Ardenas y el Boulonnais (Lethiers, 1982, 1984) y posición bioestratigráfica del yacimiento QUIN-18.

Early Frasnian correlation chart including local (Almadén) brachiopod biozonation (Pardo and García-Alcalde, 1984), the current and previous (Ziegler, 1996) conodont zonations, Ardennes and Boulonnais ostracod zonation (Lethiers, 1982, 1984) and biostratigraphic position of QUIN-18.

Svantovites inops es uno de los ostrácodos índice de la Zona DSO2 establecida por Lethiers $(1982,1984)$ en el Frasniense de las Ardenas y Boulonnais, en Francia (Fig. 4); la primera aparición de Bythocyproidea weyanti caracteriza el comienzo de la misma zona. En estas condiciones, el yacimiento QUIN-18 pertenece a la Zona DSO2, que también fue reconocida en las Cadenas Ibéricas (Gozalo, 1994).

Según la correlación sugerida por Lethiers $(1982,1984)$ (Fig. 4) la Zona DSO2 equivale a la parte alta de la Zona de $M$. asymmetricus Inferior hasta la Zona de $M$. asymmetricus Superior en la escala de conodontos (parte alta de la Zona de Palmatolepis transitans hasta la Zona de P. hassi, en la biozonación estándar).

\section{OBSERVACIONES TAFONÓMICAS}

El material del yacimiento QUIN-18 está conservado como moldes internos y externos en estratos subverticales de pelitas, más o menos finas. En el afloramiento prácticamente no aparecen superficies de estratificación expuestas. Ambos aspectos dificultan en gran medida la realización de estudios tafonómicos detallados.

Las formas más abundantes son braquiópodos, entre los cuales $P$. muelleri y $D$. alvarezi son, con mucho, las especies más frecuentes. De un total de cerca de 200 ejemplares de macrofauna identificados, $P$. muelleri representa el $41 \%$ y $D$. alvarezi el $33 \%$. En menor proporción aparecen otros braquiópodos: $C$. cf. dumontiana $(15,5 \%), R$. aff. kotalensis $(4 \%)$, y $P$. subaculeata $(3 \%)$. Completan el conjunto varias especies de bivalvos (2,5\%), trilobites (un pigidio de asteripígido) y braquiópodos inarticulados (apenas un 1\%). Hay, además, artejos de crinoideos muy pequeños (1,2 a 0,4 $\mathrm{mm}$ de diámetro) y algunos segmentos de tallos de los mismos, de hasta $5 \mathrm{~mm}$ de largo, generalmente diseminados o bien concentrados en niveles muy localizados.
Los tamaños de $P$. muelleri (anchuras entre 3,7 y 16,9 $\mathrm{mm}$ ) y $D$. alvarezi (anchuras entre 4,6 y $30,6 \mathrm{~mm}$ ) son muy variados, y se corresponden con ejemplares pertenecientes a todos los estadios de crecimiento, mientras que los ejemplares de $C$. cf. dumontiana (anchuras entre 2,8 y $8 \mathrm{~mm}$ ) son exclusivamente formas juveniles o, al menos, de pequeña talla. Las restantes formas son muy escasas y suelen ser de talla mediana dentro de la especie ( $P$. subaculeata 5,9 a $18,3 \mathrm{~mm}, R$. aff. kotalensis de 11 a $16 \mathrm{~mm}$ ), faltando las formas de mayor y menor talla.

En cuanto a porcentajes de ejemplares articulados, hay un $11,1 \%$ en $P$. muelleri, un $7,7 \%$ en $D$. alvarezi y un $3,2 \%$ en $C$. cf. dumontiana; los restantes braquiópodos aparecen siempre como moldes de valvas desarticuladas. Respecto al posible sesgo en la relación moldes de valvas dorsales a ventrales en los braquiópodos desarticulados, en todos los casos hay un porcentaje algo menor de dorsales que de ventrales; en detalle, la relación está algo más equilibrada en $P$. muelleri (56,9\% ventrales), $D$. alvarezi $(58,3 \%$ ventrales) y $R$. aff. kotalensis $(62,5 \%$ ventrales), y algo menos en $C$. cf. dumontiana $(66,7 \%$ ventrales) y $P$. subaculeata $(83,3 \%$ ventrales); de todos modos, los datos de $R$. aff. kotalensis y $P$. subaculeata están basados en muy pocos ejemplares, dada su escasez en el yacimiento.

La conservación es, en general, muy buena en $P$. muelleri, D. alvarezi, y $C$. cf. dumontiana. Por ejemplo, $P$. muelleri conserva las delicadas espinas cardinales y las finísimas arrugas superficiales y $D$. alvarezi, las frágiles orejetas, y en todos los casos se aprecian líneas de crecimiento, estrías de huellas musculares y otros sutiles detalles morfológicos, sin señales de abrasión o corrosión. Es relativamente frecuente, sin embargo (49,2\% en los ejemplares de D. alvarezi y $14,8 \%$ en los de $P$. muelleri), la presencia de perforaciones en forma de tubos radiales, paralelos a la superficie de la concha, que se inician cerca de la comisura, producidos por organismos desconocidos comparables a los que 
Racheboeuf (1981) atribuyó al género Conchifora Müller, 1968.

Como ya se indicó, las conchas originales resultaron disueltas, pero se conserva en la mayoría de los moldes una pátina de limonita, que debe provenir de la alteración de pirita diagenética, y, a veces, una película blanca, que puede corresponder a alteración hidrotermal originando pirofilita (Pardo Alonso et al., 1995).

Más problemático resulta hablar de la reorientación de los ejemplares, al haber pocas superficies de estratificación expuestas. Los moldes se disponen separados entre sí (raramente formando pavimentos densos), concordantes con la estratificación en la mayoría de los casos. Aunque en los restantes taxones se encuentran unos pocos ejemplares oblicuos o hasta perpendiculares a la estratificación, solamente en $P$. subaculeata y $R$. aff. kotalensis es frecuente esta disposición no paralela a la superficie. Por otro lado, es difícil cuantificar si hay una orientación preferente de la convexidad de los ejemplares, o de sus umbos, dadas las condiciones de afloramiento y conservación del material, pero en las muestras donde se encuentran varios ejemplares, la disposición de éstos no parece mostrar una preferencia, salvo tal vez un mayor número de ejemplares con la convexidad hacia arriba.

Es destacable la ausencia en QUIN-18 de algunos elementos faunísticos, comunes en otras zonas del Sinclinal de Almadén (sector central) y en otros sinclinales de la región (Guadalmez, principalmente), en rocas de la misma edad y de granulometría similar o más gruesa (sobre todo areniscas). Tal es el caso de Cyrtospirifer almadenensis (Paeckelmann, 1942), C. cf. almadenensis (sensu Pardo Alonso, 1997) y Apousiella bouchardi (Murchison, 1840). Estas ausencias, en todo caso, no pueden atribuirse a la destrucción por bioturbación, de la que apenas hay signos visibles.

Un primer análisis de los datos, siguiendo las indicaciones de varios autores, compendiadas por Speyer y Brett (1988), sería el siguiente:

La abundancia y buena conservación de los ejemplares, el alto porcentaje de moldes de conchas articuladas, el escaso sesgo de tamaños y la relación equilibrada de moldes de valvas dorsales a ventrales, así como la disposición en la roca y la finura del sedimento original con relación al tamaño de los ejemplares indican que $P$. muelleri y $D$. alvarezi serían formas autóctonas o parautóctonas, que vivieron sobre un sedimento blando, de grano muy fino, sometido a débiles corrientes; la velocidad de sedimentación debió de ser lo suficientemente rápida como para conservar muchos ejemplares articulados y evitar la corrosión por exposición prolongada de las conchas pero, a la vez, lo suficientemente lenta como para permitir que otros ejemplares fuesen desarticulados y colonizados por organismos perforantes. Las dos especies presentan formas bastante aplanadas, adaptadas a la vida sobre sedimentos blandos (ver apartado de Paleoecología).

En cuanto a las restantes formas, los sesgos de tamaño, relación entre moldes de valvas dorsales a ventrales, fragmentación, etc. parecen indicativos de un origen alóctono, dudoso quizá en el caso de $C$. cf. dumontiana. Los elementos alóctonos serían transportados desde zonas de mayor energía (posiblemente más someras), ya que los braquiópodos articulados son más frecuentes en rocas de grano más grueso, principalmente areniscas, que se encuentran a una altura estratigráfica similar (Fm. Tres Mojones) en el Sinclinal de Guadalmez; la disposición frecuente no paralela a la superficie de estratificación de algunas de estas formas, en ausencia de bioturbación y oleaje, parece indicar episodios de transporte en masa. La llegada de estos elementos exóticos pudo deberse a tormentas fuertes, que parecen indicadas también por la presencia de niveles limolíticos o areniscosos milimétricos, conteniendo pequeños artejos de crinoideos y otros bioclastos no identificables; esta sedimentación episódica sería más rápida que la habitual en el área.

Los ejemplares de $C$. cf. dumontiana podrían ser también formas alóctonas, arrastradas por corrientes capaces de seleccionar las formas de menor talla, o bien (dada su relativa abundancia, buena conservación, menor sesgó en la relación de moldes de valvas dorsales a ventrales y desarticulación) formas autóctonas o parautóctonas, cuyo tamaño, menor que el habitual, sería debido a condiciones de vida relativamente desfavorables.

Los ostrácodos permiten también interesantes inferencias tafonómicas. De acuerdo con las observaciones de Pokorny (1965) y Oertli (1971), un primer carácter a considerar es la ratio entre moldes de valvas aisladas y de caparazones. En este sentido, la presencia de más de un $30 \%$ de moldes de caparazones completos en el yacimiento QUIN-18 habla en favor de condiciones de sedimentación relativamente rápidas en un substrato poco consolidado. Por otro lado, la disposición de los moldes de valvas aisladas y caparazones en este yacimiento es similar al tipo A, de la figura 7 de Guernet y Lethiers (1989), con los moldes de las valvas aisladas subparalelos a la superficie de estratificación y los de los caparazones completos oblicuos a ella, lo que representaría ambientes de poca energía, con velocidad de sedimentación alta, substratos poco consolidados y bioturbación escasa o nula.

Otro aspecto interesante es el que hace referencia a la estructura poblacional de los ostrácodos bentónicos, es decir, la presencia o ausencia de los distintos estadios ontogenéticos, para deducir la autoctonía o aloctonía de las asociaciones registradas (Whatley, 1983, 1988). Aunque, dado el pequeño tamaño de la muestra disponible (57 ejemplares), no es posible hablar en detalle de la estructura poblacional, la presencia casi exclusiva en ella de ejemplares adultos permitiría encuadrar esta asociación en la distribución de tipo B propuesta por Whatley (ops. cits.). Este autor interpreta que dicha distribución representa bien una biocenosis de alta energía o bien una tanatocenosis de la que las corrientes eliminaron las formas embrionarias más pequeñas. La primera de estas interpretaciones es claramente contradictoria con los datos deducidos previamente, mientras que la segunda indicaría una 
selección de tamaño por transporte y, por lo tanto, el carácter alóctono de la asociación, que, en el caso de la muestra estudiada, procedería de algún punto cercano, quizás de parches arrecifales presentes en el CVS de Chillón.

Con todas las reservas debidas al tipo de afloramiento y tamaño de la muestra, la tafofacies de QUIN-18 es similar a algunas de las que Speyer y Brett (1991) describieron en el Grupo Hamilton (Devónico Medio del Estado de Nueva York), en especial a la tafofacies VII (dysaerobic, sediment-dominated beds, "barren shales"), aunque existen algunas diferencias interesantes. Dicha tafofacies se desarrollaría en el área de mayor profundidad dentro de un mar epicontinental, con corrientes de baja energía y tasa de sedimentación moderada a alta. La mayor discrepancia se encuentra en la constante presencia en la tafofacies norteamericana de formas pelágicas (estiliolinas, cefalópodos, etc.), que no aparecen en QUIN-18. En la misma línea, es notable también la ausencia de entomozoidos, entre los ostrácodos. Una posible explicación de esta anomalía sería el aislamiento del área, debido a la presencia de una barrera que impediría la comunicación con el mar abierto, a la que ya aludimos, constituida por los edificios volcánicos desarrollados hacia el oeste (Fig. 3B).

Se trataría, en definitiva, de un nuevo modelo de tafofacies asociado a un medio similar al del modelo VII de Speyer y Brett (1991), pero que se desarrollaría en condiciones protegidas, relativamente restringidas.

\section{PALEOECOLOGÍA}

Por lo que respecta a los braquiópodos, P. muelleri y D. alvarezi serían formas autóctonas, como ya se indicó antes. De acuerdo con Racheboeuf (1981), la forma cóncavo-convexa, la obstrucción del foramen por placas (ausencia de pedúnculo, al menos en estado adulto) y la presencia de espinas cardinales en los chonétidos serían indicativos de formas de vida libre, apoyadas en fondos fangosos, no consolidados, sobre la valva ventral, cumpliendo las espinas una función de sujeción extra que evitaría el hundimiento de las conchas por su parte posterior, más pesada conforme se produce el crecimiento. En detalle, $P$. muelleri es un chonétido de pequeña talla y concha fina, débilmente cóncavoconvexo, con un número moderado de espinas cardinales (hasta 3 en cada lado), más o menos largas, de tipo ortomorfo recto a algo oblicuo; estas características, de acuerdo con Racheboeuf (1990), indicarían un modo de vida libre. El organismo viviría reposando sobre un substrato blando, o parcialmente hundido en él, en un medio profundo o en medios más someros, pero tranquilos, de carácter restringido, con las espinas ejerciendo una función de anclaje.

La morfología de $D$. alvarezi se aproxima mucho a la que Thayer (1975) atribuye a adaptaciones a substratos blandos. En efecto, el perfil plano-convexo, la delgadez de la concha, la presencia de seudodeltidio (que obtura el foramen), y la gran superficie externa en comparación con el volumen de la cavidad interior (que conferiría una mayor «flotabilidad» a las conchas), abogan porque fueran formas de vida libre, que yacerían sobre fondos blandos, probablemente sobre su valva ventral.

La baja diversidad y el escaso número de formas autóctonas y de señales de bioturbación, frente a las condiciones opuestas que se registran en otras partes del Sinclinal de Almadén, en niveles de la misma o parecida edad, indican, de nuevo, un medio restringido y estresante en QUIN-18, quizás debido a la profundidad, al tipo de substrato, muy blando, o al bajo nivel de oxígeno, o a combinaciones de dichos factores. No obstante, sería necesario un estudio más amplio y completo de los niveles contemporáneos, de condiciones más abiertas, para llegar a conocer con precisión tales extremos.

Como ya se indicó, aparte de D. alvarezi, P. muelleri y, quizás, de $C$. cf. dumontiana, los braquiópodos provendrían de medios más agitados, por encima del nivel de base del oleaje de tormentas, ya que son elementos que suelen encontrarse también en otras áreas del sinclinal, en rocas de grano más grueso (limolitas arenosas, areniscas y areniscas cuarcíticas), con intenso retrabajamiento, fragmentación, selección y concentración de bioclastos. Es probable que estas áreas estén representadas, como se ha sugerido, por los conos volcánicos situados hacia el oeste, desarrollados en condiciones más someras, por acumulación de piroclastos y coladas de lava sobre el fondo marino.

Respecto a los ostrácodos, se han propuesto varios modelos de asociaciones en el Devónico para representar los distintos ambientes sedimentarios. De acuerdo con la propuesta de división de Becker (en Bandel y Becker, 1975), los ostrácodos de QUIN-18 pertenecerían al ecotipo (ökotyp) eifélico, subtipo vorrif (pre-arrecifal) de Becker (1981), caracterizado por aguas someras y agitadas, normalmente por encima del nivel de acción de las olas. Siguiendo a Guernet y Lethiers (1989), la asociación estudiada podría compararse con el grupo 8 de asociaciones del Paleotetis, las denominadas «associations péri- et subrécifales au Frasnien». Es decir, ambos modelos llevan a interpretar los ostrácodos de QUIN-18 como propios de un ambiente peri-arrecifal, sublitoral, de alta energía. Estos datos son, en principio, contradictorios con los inferidos a partir de los braquiópodos y con las observaciones tafonómicas y sedimentológicas realizadas. La aparente contradicción se resolvería si, como ya se repitió varias veces, los ostrácodos representasen una fauna alóctona, arrastrada desde ambientes peri-arrecifales hasta el yacimiento QUIN-18, de manera fortuita, por ejemplo en un período tormentoso fuerte, igual que los elementos macrofaunísticos analizados. Esta suposición viene avalada por la presencia, en las laderas de los aparatos volcánicos submarinos contemporáneos, de pequeños cuerpos arrecifales (Fig. 3), con asociaciones de ostrácodos similares a la de QUIN-18 (Pardo y García-Alcalde, 1984, p. 86: yacimiento V-960). 

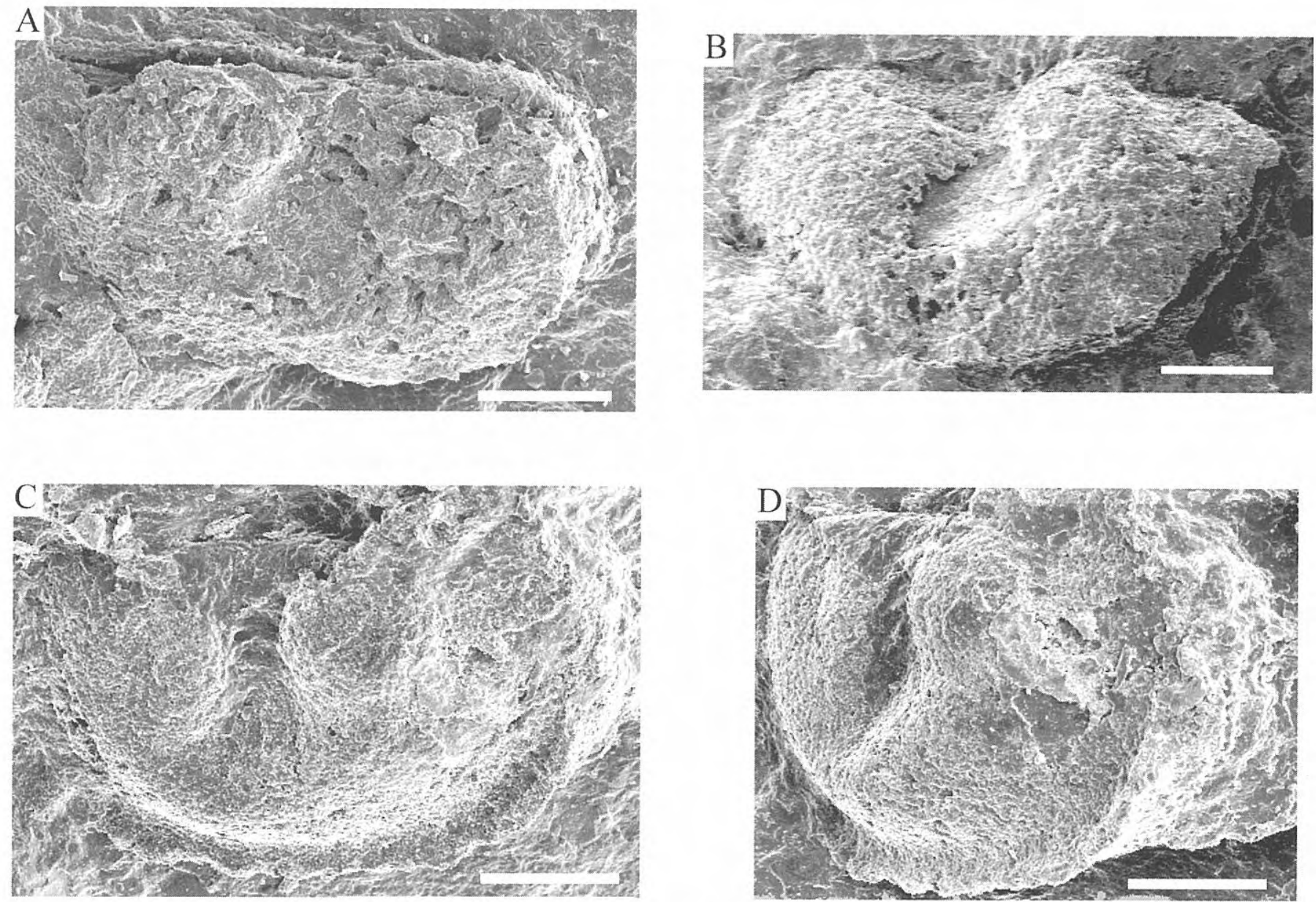

Figura 5.Hollinella (Keslingella) ? sp. Escala $200 \mu \mathrm{m}$. A.- Vista anterolateral de molde en látex de valva derecha de un caparazón (ejemplar MGUV-2899a). B.- Vista posterolateral de molde en látex de la valva izquierda del caparazón anterior. C.- Vista lateral de molde en látex de la valva izquierda de un caparazón (ejemplar MGUV-7561). D.- Vista oblicua posterior del ejemplar anterior.

Bar scale $200 \mu \mathrm{m}$. A.- lateral-anterior view of a shell right valve, latex cast (specimen MGUV-2899a). B.- Lateralposterior view of the left valve of the same shell, latex cast. C.- Lateral view of a left shell, latex cast (specimen $M G U V-7561)$. D.- Oblique-posterior view of $C$.

\section{SISTEMÁTICA}

Como ya se comentó, los fósiles son moldes internos y externos, sin resto de la concha original. Entre los ostrácodos, esto puede representar un importante problema, porque sólo son posibles buenas aproximaciones taxonómicas en formas con ornamentación o diseño de las valvas muy característicos, mientras que las que requieren un análisis completo del caparazón y de la articulación no pueden determinarse a niveles específicos. En este apartado se aportan también nuevos datos taxonómicos, paleogeográficos y estratigráficos de la forma más frecuente de braquiópodos, Pradochonetes muelleri.

El material figurado y/o medido se encuentra depositado en el Museo de Geología de la Universitat de València con las siglas MGUV. Una pequeña parte del material de $P$. muelleri procede de la colección del Museo Geominero de Madrid (siglas MGM).
SUBCLASE OSTRACODA Latreille, 1802 ORDEN PALAEOCOPIDA Henningsmoen, 1953 SUBORDEN HOLLINOMORPHA Henningmoen, 1965 Superfamilia Hollinoidea Swartz, 1936

Familia Hollinellidae Bless y Jordan, 1971 Género Hollinella Coryell, 1928

Subgénero Hollinella (Keslingella) Bless y Jordan, 1970

Especie tipo: Hollinella pumila Kesling, 1952.

\section{Hollinella (Keslingella)? sp. \\ Fig. 5}

Material: Tres moldes de caparazones y otros tres de valvas aisladas.

\section{Discusión}

Los ejemplares son de tipo bilobado complejo (sensu Bless y Jordan, 1971), con una estructura adventral tipo 
velum (que no se encuentra incurvado); estas características permiten clasificar los especímenes como pertenecientes al género Hollinella. La estructura y forma del velum de los ejemplares encontrados son claramente diferentes de las estructuras velares de los subgéneros $H$. (Praehollinella) Bless y Jordan, 1971 y H. (Truyolsella) Sánchez de Posada y Bless, 1999. Por otro lado, todos los ejemplares encontrados son adultos y la diferenciación entre los subgéneros $H$. (Hollinella) y $H$. (Keslingella) se realiza de acuerdo con la morfología de las formas juveniles, por lo que la asignación subgenérica presenta dudas. En cualquier caso, hay que recordar que hasta el momento en el Frasniense sólo se citaron formas de $H$. (Keslingella), mientras que H. (Hollinella) parece propio del Carbonífero y Pérmico (Bless y Jordan, 1970, 1971), por lo que tentativamente asignamos nuestro material al subgénero $H$. (Keslingella).

ORDEN METACOPINA Sylvester-Bradley, 1961

Superfamilia Healdioidea Harlton, 1933

Familia Healdiidae Harlton, 1933

Género Bythocyproidea Stewart y Hendrix, 1945

Especie tipo: Bythocyproidea sanduskyensis Stewart y Hendrix, 1945.

\section{Bythocyproidea weyanti (Becker, 1971) Fig. 6}

- 1989 Punctomosea weyanti Becker, 1971; Casier, lám. 1: 17.

v 1994 Bythocyproidea weyanti (Becker, 1971); Gozalo, 91-93, lám. 13: 2-8 (con sinónimos anteriores).

Material: Seis moldes de valvas, tres en estado fragmentario.

\section{Discusión}

Una buena descripción de la especie se encuentra en los trabajos de Becker (1971) y Gozalo (1994). Estos autores describieron un amplio rango de variación morfológica para Bythocyproidea weyanti. Estas variaciones morfológicas se observaron en ejemplares registrados en el mismo nivel, e incluyen, entre otros aspectos, variación del contorno del caparazón, distinto desarrollo de la superficie ornamentada y presencia o ausencia de crestas y espinas posteriores. Los ejemplares del Sinclinal de Almadén presentan una morfología similar a los especímenes previamente asignados a la especie.

\section{Distribución estratigráfica y yacimientos}

La especie se citó previamente en las cuencas de Dinant y Namur, en Bélgica (Becker, 1971; Lethiers, 1974, 1982; Casier, 1977, 1987, 1989) y en las Cadenas Ibéricas (Gozalo, 1994). La distribución bioestratigráfica en las cuencas de Dinant y Namur sería, según Lethiers (1982, 1984), desde la Zona DSO2 a la DSO5 (FrasnienseFameniense Inferior), equivalente a la sugerida por Casier
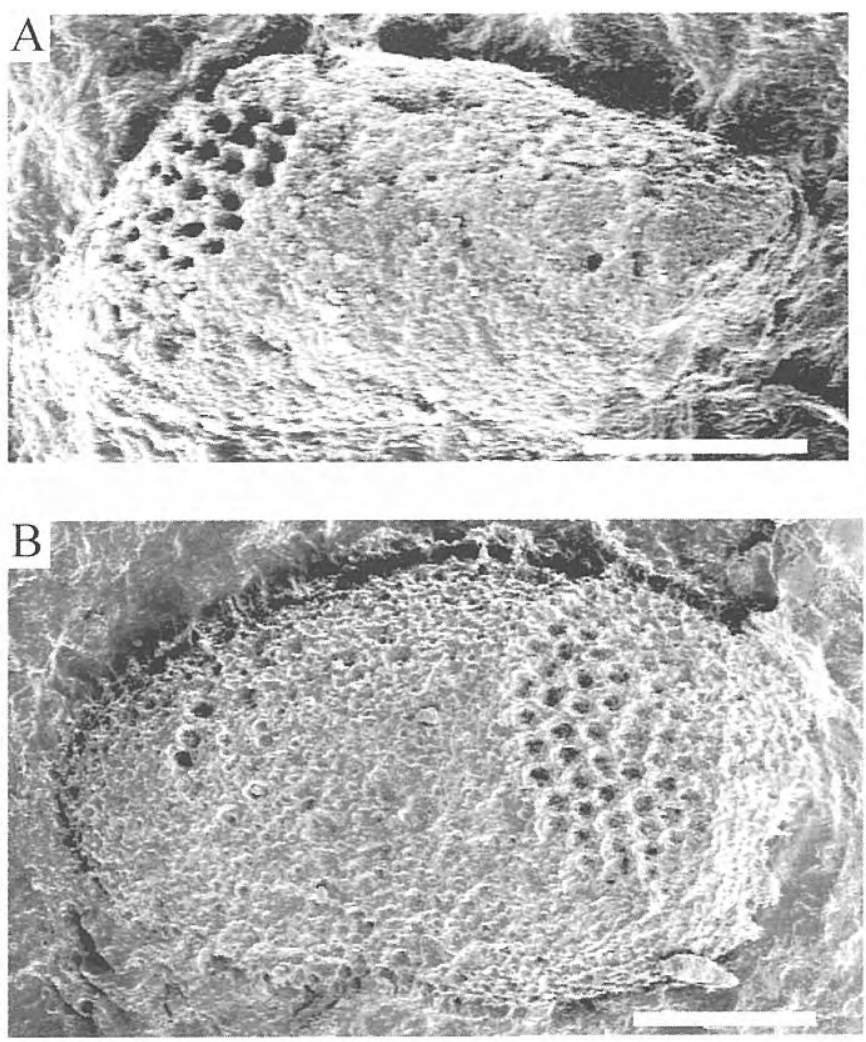

Figura 6. Bythocyproidea weyanti (Becker, 1971). Escala $250 \mu \mathrm{m}$. A.- Molde en látex de valva derecha (ejemplar MGUV-2898). B.- Molde en látex de valva izquierda (ejemplar MGUV-2897).

Bar scale $250 \mu \mathrm{m}$. A.- Right valve latex cast (specimen MGUV-2898). B.- Left valve latex cast (specimen $M G U V$-2897).

(1977, 1987), que abarcaría las Zonas de Favulella lecomptei y Svantovites lethiersi.
Superfamilia Thlipsuroidea Ulrich, 1894 Familia Thlipsuridae Ulrich, 1894
Género Polyzygia Gürich, 1896

Especie tipo: Polyzygia symmetrica Gürich, 1896.

\section{Polyzygia neodevonica neodevonica (Matern, 1929) \\ Fig. 7}

? 1984 Polyzygia cf. neodevonica (Matern); Pardo y García-Alcalde, 86.

- 1989 Polyzygia neodevonica (Matern, 1929); Casier, lám. 1: 19.

- 1993 Polyzygia neodevonica (Matern 1929); Lethiers y Racheboeuf, 598, lám. 2: 7-8.

v 1994 Polyzygia neodevonica neodevonica (Matern, 1929); Gozalo, 100-102, lám. 15: 4-5 (con sinónimos anteriores).

- 1995 Polyzygia neodevonica (Matern 1929); Becker, 139, lám. 1: 1-2. 

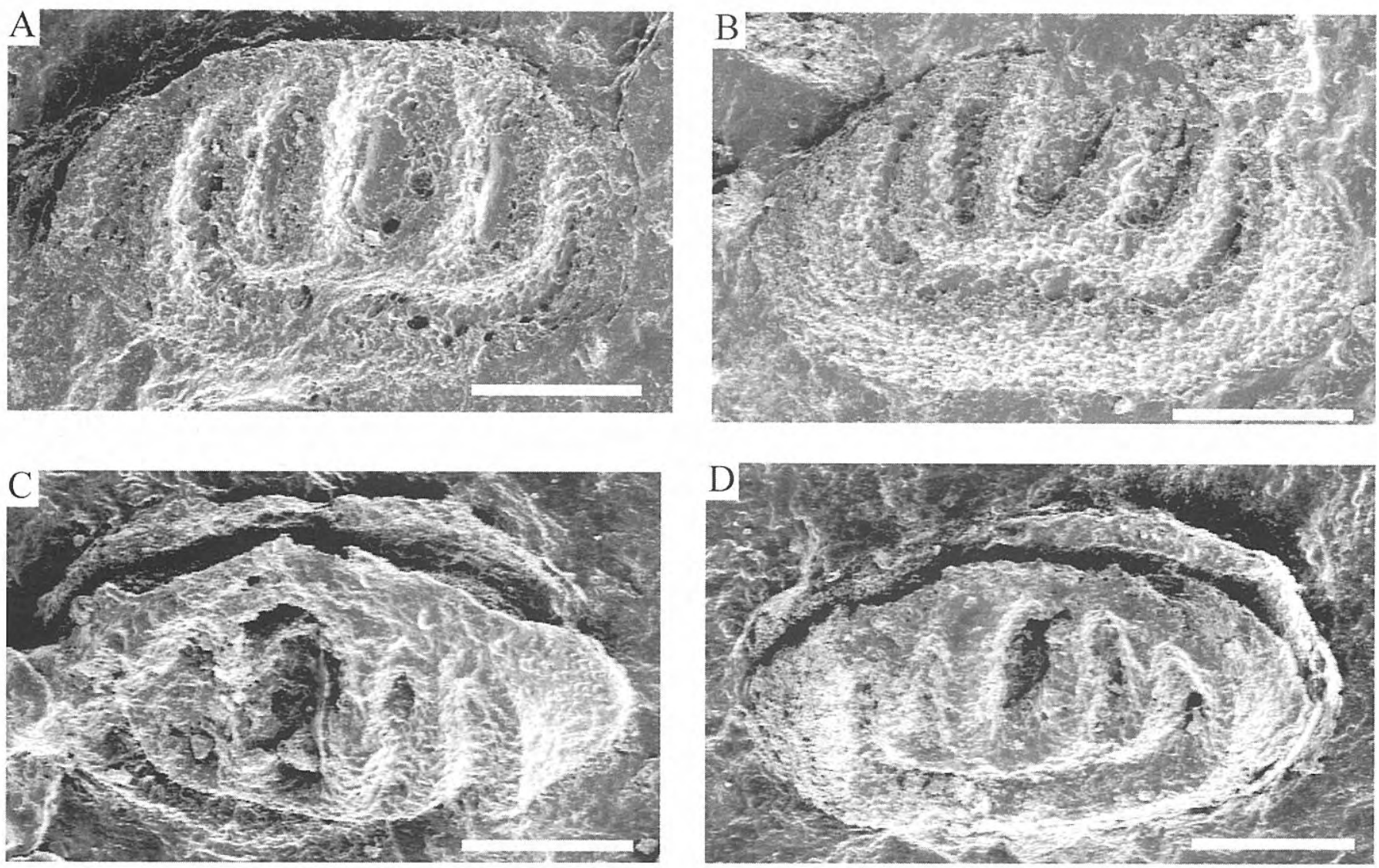

Figura 7. Polyzygia neodevonica neodevonica (Matern, 1929). Escala $250 \mu \mathrm{m}$. A.- Vista lateral de un molde en látex de una valva izquierda (ejemplar MGUV-7562). B.- Vista lateral de un molde de látex de una valva izquierda (ejemplar MGUV-2894a). C.- Molde en látex de un caparazón, vista dorsal inclinada y valva derecha (ejemplar MGUV-2896). D.- Molde en látex de un caparazón, vista dorsal inclinada y valva izquierda (ejemplar MGUV-2895a).

Bar scale $250 \mu \mathrm{m}$. A.- Lateral view of left valve latex cast (specimen MGUV-7562). B.- Idem (specimen MGUV2894a). C.- Tilted dorsal view of a right valve latex cast (specimen MGUV-2896). D.- Tilted lateral view of a left valve latex cast (specimen MGUV-2895a).

- 2000 Polyzygia neodevonica (Matern 1929); Becker, 582-583.

Material: Seis moldes de caparazones completos y diez de valvas aisladas.

\section{Discusión}

Los ejemplares del yacimiento QUIN-18 son formas adultas, salvo dos ejemplares que representan el último estadio juvenil (A1), con el surco ventral atenuado. La surcación de la superficie lateral permite incluirlos en la especie Polyzygia neodevonica, mientras que la profundidad de los surcos, la forma de las valvas y la ausencia de espinas posteriores los asimila a $P$. neodevonica neodevonica, distinguiéndose claramente de $P$. neodevonica aragonensis Gozalo y Sánchez de Posada, 1986. En conjunto, la morfología del nuevo material encaja en el rango de variabilidad de la subespecie, descrito por Becker (1971) y Michel (1972).

\section{Distribución estratigráfica y yacimientos}

La subespecie presenta una amplia distribución geográfica, habiéndose citado previamente en el Macizo
Armoricano, el Boulonnais, las Ardenas, las Montañas del Rín, la Cordillera Cantábrica, las Cadenas Ibéricas, la Zona Centroibérica y Argelia. Su distribución estratigráfica abarca desde el Givetiense Medio al Fameniense Inferior (ver Becker, 1988, 2000).

Familia Quasillitidae Coryell y Malkin, 1936 Género Ponderodictya Coryell y Malkin, 1936

Especie tipo: Leperditia punctulifera Hall,1860.

\section{Ponderodictya cf. belliloci Casier, 1986 Fig. 8}

Material: Dos moldes de caparazones y seis de valvas aisladas.

\section{Discusión}

El material es asimilable a Ponderodictya belliloci, tanto por el tamaño de los ejemplares como por el contorno de las valvas y ornamentación, pero por un lado 

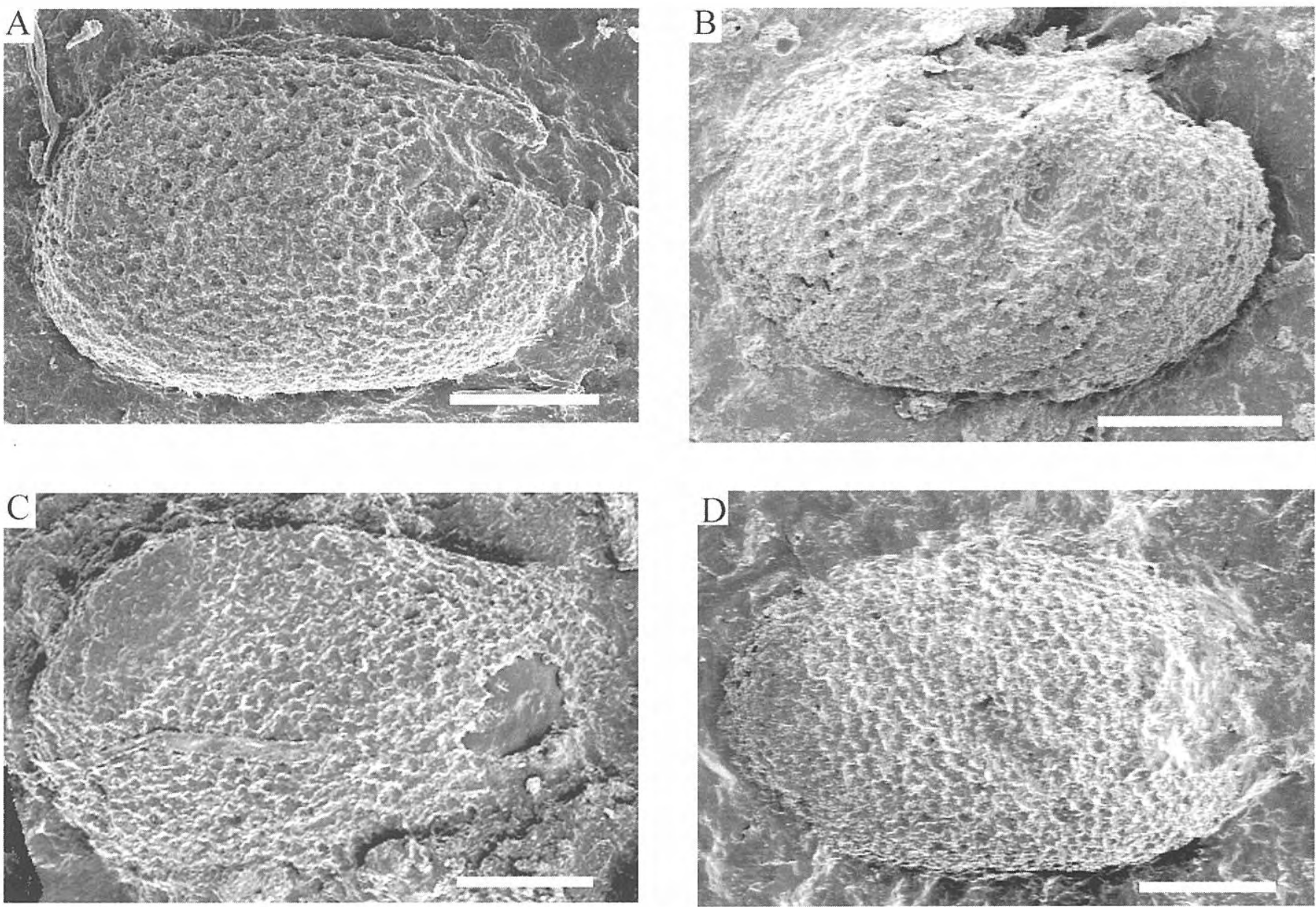

Figura 8. Ponderodictya cf. belliloci Casier, 1986. Escala $200 \mu \mathrm{m}$. A.- Molde en látex de valva izquierda (ejemplar MGUV2899b). B.- Molde en látex de caparazón, vista lateral izquierda (ejemplar MGUV-2894b). C.- Molde en látex de valva derecha (ejemplar MGUV-7563). D.- Molde en látex de valva izquierda (ejemplar MGUV-2894c).

Bar scale $200 \mu m$. A.- Lateral view of left valve latex cast (specimen MGUV-2899b). B.- Left lateral shell view of latex cast (specimen MGUV-2894b). C.- Right valve view of latex cast (specimen MGUV-7563). D.- Left valve view of latex cast (specimen $M G U V-2894$ c).

la falta de caparazones completos que permitan establecer de manera precisa la relación entre ambas valvas, y por otro la ausencia de la pequeña espina posteroventral habitual en la especie, aunque no siempre se desarrolle (Lethiers et al., 1985; Casier, 1986), hacen preferible mantener los ejemplares estudiados en nomenclatura abierta. Otra especie que presenta una cierta similitud con el material estudiado es P. ostententatia Loranger, 1963, del Frasniense de Alberta (Canada), pero su ornamentación es mucho más fina y el diseño de la reticulación de menor tamaño.

\section{Distribución estratigráfica y yacimientos}

Ponderodictya belliloci fue citada en varios yacimientos de las cuencas de Dinant y de Namur, siempre en materiales frasnienses, concretamente desde la base de la Zona de $M$. asymmetricus Inferior hasta la parte media de la Zona de $M$. asymmetricus Superior de conodontos (Casier, 1986). Posteriormente Becker (1988, 1995) la citó en materiales del Givetiense Superior de la Cordillera Cantábrica.
Género Svantovites Pokorný, 1951

Especie tipo: Svantovites primus Pokorný, 1951.

\section{Svantovites inops Becker, 1971 Fig. 9}

v • 1994 Svantovites inops Becker, 1971; Gozalo, 117-118, lám. 19: 1-2 (con sinónimos anteriores).

Material: Dos moldes de valvas izquierdas, una de ellas en estado framentario.

\section{Discusión}

El único molde completo de valva izquierda del yacimiento QUIN-18 presenta la superficie lateral con ornamentación constituida por 11 costillas subverticales, de diseño idéntico al de los tipos de la especie (Becker, 1971: lám. 10, figs. 94-96). Descripciones de $S$. inops han sido publicadas en los trabajos de Becker (1971) y Gozalo (1994). 


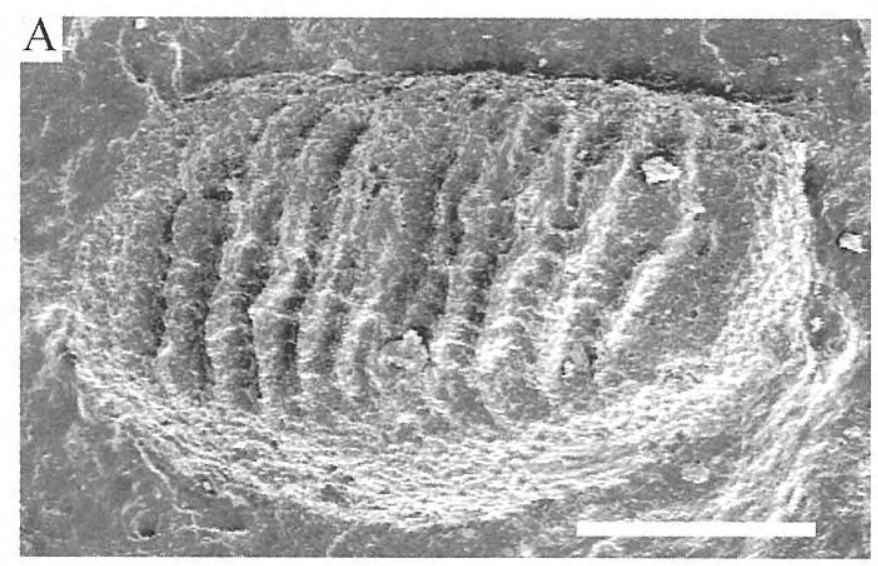

Figura 9.Svantovites inops Becker, 1971. Escala $250 \mu \mathrm{m}$. A.- Molde en látex de valva izquierda (ejemplar MGUV-2895b), vista lateral ligeramente inclinada. Bar scale $250 \mu \mathrm{m}$. A.- Slightly tilted lateral view of left valve latex cast (specimen MGUV-2895b).

\section{Distribución estratigráfica y yacimientos}

La especie se citó en las Ardenas (Becker, 1971; Lethiers, 1982, 1984; Casier, 1987), en el Boulonnais (Lethiers, 1982, 1984; Lethiers et al., 1985) y en las Cadenas Ibéricas (Gozalo, 1994). Su distribución ha sido utilizada por Lethiers (1982, 1984), junto con la de otras formas, para caracterizar su Zona DSO2.

PHYLUM BRACHIOPODA Duméril, 1806 SUBPHYLUM RHYNCHONELLIFORMEA Williams et al., 1996

CLASE STROPHOMENATA Williams et al., 1996

ORDEN PRODUCTIDA Sarytcheva y Sokolskaya, 1959

SUBORDEN CHONETIDINA Muir-Wood, 1955

Superfamilia Chonetoidea Bronn, 1862

Familia Chonetidae Bronn, 1862

Subfamilia Dagnachonetinae Racheboeuf, 1981

Género Pradochonetes Pardo y García-Alcalde, 1984

Especie tipo: Pradochonetes muelleri Pardo y GarcíaAlcalde, 1984.

Pradochonetes muelleri

Pardo y García-Alcalde, 1984

Fig. 10

v* 1984 Pradochonetes muelleri n. sp.; Pardo y GarcíaAlcalde, 105-106, 108-109, fig. 9 d-t. 5 (con sinónimos anteriores).

Ø 1986 Pradochonetes muelleri García-Alcalde, 1984; Racheboeuf, en Racheboeuf et al., 40, lám. 1, fgs. 4-8.

v 1987 Pradochonetes muelleri; Pardo Alonso y GarcíaAlcalde, en García Sansegundo et al., 27.

v 1989 Pradochonetes muelleri Pardo y García-Alcalde; Pardo Alonso y García-Alcalde, en Olivé Davó et al., 50.

v 1992 Pradochonetes muelleri Pardo y García-Alcalde; García-Alcalde, en Soldevila Bartolí, 372.

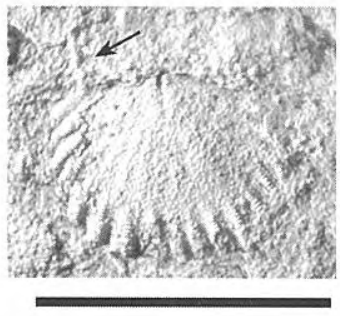

A

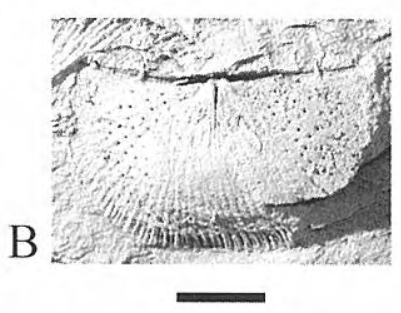

C

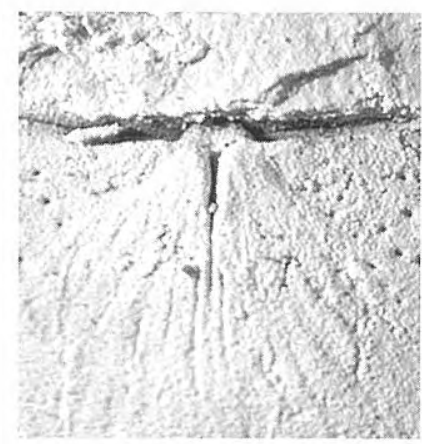

$\mathrm{D}$

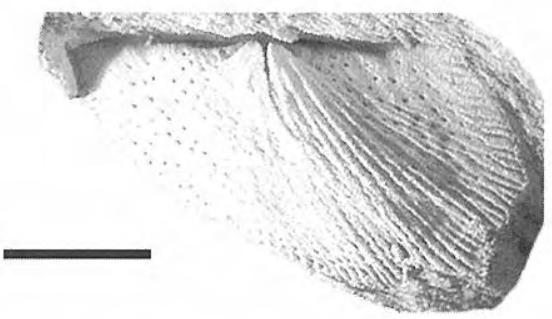

Figura 10. Pradochonetes muelleri Pardo y García-Alcalde, 1984. Escala $3 \mathrm{~mm}$. A.- Molde interno ventral de un ejemplar muy joven, con una espina cardinal (flecha) bien conservada (ejemplar MGUV 1510-2). B.- Molde interno de una valva ventral de un ejemplar geróntico, mostrando al menos 5 espinas cardinales, y las corrugaciones bien conservadas (ejemplar MGUV-1500-1). C.Detalle de la región cardinal del ejemplar anterior, con el campo muscular y el relleno del canal interno de la espina cardinal más próxima al umbo. D.- Molde interno ventral deformado, con varias espinas cardinales; la deformación no es tectónica sino por compactación del sedimento pelítico en un ejemplar no paralelo a la estratificación (ejemplar MGUV-1509-1).

Bar scale $3 \mathrm{~mm}$. A.- Inner ventral cast of a very juvenile specimen, with very well preserved cardinal spine (arrow) (specimen MGUV-15102). B.- Inner ventral cast of a gerontic specimen showing at least 5 cardinal spines and well preserved corrugations (specimen MGUV-15001). C.- Detail of specimen B, with muscle scar and inner channel filling of the cardinal spine closest to the umbo. D.- Deformed inner ventral cast with several cardinal spines; deformation is not tectonic but created by pelitic sediment compaction of an specimen non parallel to bedding (specimen MGUV-1509-1). 
v 1994 Pradochonetes muelleri; Pardo Alonso y GarcíaAlcalde, fig. 2.

v 1996 Pradochonetes muelleri; Pardo Alonso y GarcíaAlcalde, 77, fig. 3.

v 1999 Pradochonetes muelleri Pardo \& García-Alcalde 1984; Pardo Alonso, lám. 2: 23.

Material y yacimientos: En el yacimiento QUIN-18 (sinclinal de Almadén) ejemplares MGUV-1500, MGUV1501-2, MGUV-1506-2, MGUV-1509-1, MGUV-1509-2, MGUV-1510-2, MGUV-1510-3, MGUV-1510-4, MGUV1511, MGUV-1512, MGUV-2099. (MGUV-1500-1, MGUV 1510-2 y MGUV-1509-1, se figuran aquí, Fig. 10).

Además de este material y del consignado en la descripción original de la especie, se han hallado nuevos ejemplares procedentes, en la mayor parte de los casos, de yacimientos hasta ahora inéditos:

a) Sinclinal de Herrera. MGUV-2071-1, MGUV-2071-2, MGUV-2071-3, MGUV-2071-4, MGUV-2071-6, MGUV2071-7, MGUV-2071-8, MGUV-2071-9, MGUV-2077-1, MGUV-2077-2, MGUV-2095-3, MGUV-2095-4 (yacimiento CS-1); MGUV-1168-4 (yacimiento $\mathrm{PH}-2$ ).

b) Sinclinal de Almadén. MGM-234D-2 (localidad "Chillón”, según indica la etiqueta); MGUV-1154-2 (yacimiento V-102).

c) Sinclinal de Guadalmez. MGUV-693-4 (yacimiento NG1).

A estos hay que añadir nuevos yacimientos localizados en Pardo Alonso (1997), con material no siglado: EP-22, EP-23, EP-25, PH-3 y VMY-25; G-14, GC-1, GC-2, GO-2, SG-16, TRM-15, TRM-16, TRM-19, TRM-1s, TRM-22, VS-1s y VS-4s (Sinclinal de Guadalmez); CBU-7s (flanco norte del Sinclinal de Pedroches, en Cabeza del Buey).

\section{Discusión}

Forma común en los niveles principalmente pelíticos de la parte media y alta del Frasniense de la región de Almadén. Es más frecuente en los niveles pelíticos, aunque también se encuentra puntualmente en las unidades cuarcíticas; por su forma característica, de fácil identificación, y por tratarse del único chonétido que se ha encontrado hasta el presente en estos niveles, resulta muy útil como herramienta en correlaciones locales.

Racheboeuf (en Racheboeuf et al., 1986) asignó a $P$. muelleri ejemplares procedentes del Frasniense de la cantera de Alange (Badajoz); sin embargo, de acuerdo con las figuras y la descripción aportados por el autor, las formas de Alange son netamente más pequeñas y menos transversas, con dos espinas a cada lado de la charnela (hasta tres a cada lado, en P. muelleri, fig $10 \mathrm{~B}$ ); los ángulos cardinales son rectos, mientras en nuestra especie suelen prologarse en débiles orejetas, y no se observa la suave depresión media ventral típica en nuestras formas. En el interior ventral, las improntas musculares quedan reducidas a los aductores, separadas por un miofragma corto y robusto, mientras en $P$. muelleri, el miofragma es largo, sobre 1/3 de la longitud de la valva (Fig 10 B y C), y hasta $1 / 2$ en algún ejemplar, y es posible separar con claridad el campo de los diductores, especialmente en sus límites posterolaterales que están excavados; en el interior dorsal, es muy evidente la práctica ausencia de brevisepto en el material de Alange, mientras que en nuestra especie es característicamente fuerte y largo, bien desarrollado incluso en especímenes de talla comparable a los de Alange. Otros caracteres típicos de Pradochonetes muelleri, como la forma trilobada de la apófisis cardinal, no se mencionan ni distinguen en la figuración de Racheboeuf. Por todo ello, separamos las formas de Alange de P. muelleri, especialmente por las diferencias notables en el interior de ambas valvas, que incluso la apartarían del propio género Pradochonetes.

\section{Distribución estratigráfica y geográfica}

Con los nuevos hallazgos, la distribución estratigráfica de la especie abarca del miembro superior de la Fm. Valmayor, donde es muy frecuente en toda la región estudiada, a la parte media de la Fm. Valdegregorio. Aunque más escasa, se encuentra también en las areniscas y calizas arenosas bioclásticas que constituyen la Fm. Tres Mojones; más raramente aparece en niveles arenosos dentro de la Fm. Valdegregorio, donde la encontramos hasta el tercio superior de la unidad en Guadalmez y en el Sinclinal de Herrera del Duque (sección de Valmayor-II de Pardo Alonso, 1997).

Caracteriza la Biozona de Extensión de P. muelleri, la más alta de las definidas en el Frasniense en esta parte de la Zona Centroibérica. Se encontró por primera vez en la terminación oriental del sinclinal de Almadén, en el área de Cabeza del Buey, y también en Herrera del Duque; esto último supone que el material citado por Puschmann (1970) como Chonetes armatus, procedente de la Fm. Valmayor, casi con toda seguridad pertenece a $P$. muelleri. Su extensión geográfica, en la rama meridional de la Zona Centroibérica, comprende desde el flanco norte del Sinclinal de Pedroches hasta el de Herrera del Duque. Una cita puntual se encuentra en Soldevila Bartolí (1992, determinación de J.L. GarcíaAlcalde) en material procedente de la unidad de La Víbora (localidad ABQ-87); esto amplía su distribución geográfica hasta la Sierra de San Pedro.

\section{AGRADECIMIENTOS}

Los autores agradecen a los Dres. Jenaro García-Alcalde, Luis C. Sánchez Posada y María Luisa Martínez Chacón su revisión crítica al manuscrito que ha mejorado el texto original. A la Dra. Isabel Rábano, directora del Museo Geominero (IGME, Madrid), le agradecemos el préstamo de los ejemplares de braquiópodos devónicos guardados en esa institución. Este trabajo es una contribución a los Proyectos PB98-1542 y PB98-1558 financiados por la Dirección General de Estudios Superiores, y también al proyecto I.G.C.P. $\mathrm{n}^{\circ} 421$ North Gondwana Mid-Palaeozoic Bioevent/ Biogeography patterns in relation to crustal dynamics.

\section{BIBLIOGRAFÍA}

Bandel, K. und Becker, G. 1975. Ostracoden aus paläozoischen pelagischen Kalken der Karnischen Alpen (Silurium bis Unterkarbon). Senckenbergiana lethaea, 56, 1-83. 
Becker, G. 1971. Ostracoda aus dem Mittel-Frasnium (Oberdevon) der Mulde von Dinant. Bulletin de l'Institut Royal des Sciences naturelles de Belgique, Sciences de la Terre, 47, 1-82.

Becker, G. 1981. Ostracoden im Devon Europas palaökologische, palaeogeographische und biostratigraphische Aspekten. Natur und Museum, 111, 378-383.

Becker, G. 1988. Neritische Ostracoden aus den HuergasFormation des Kantabrischen Gebirges (Givetium; NSpanien). Senckenbergiana lethaea, 68, 393-431.

Becker, G. 1995. Benthische Ostracoden aus dem PräeFamennium des Kantabrischen Gebirges (Devon; NSpanien). Senckenbergiana lethaea, 75, 131-148.

Becker, G. 2000. Zur Chronologie paläozoischer Ostracoden. Eine Bestandsaufnahme für das Mitteldevon der Eifel. Senckenbergiana lethaea, 79, 569-588.

Bless, M. J. M. and Jordan, H. 1970. Stratigraphical and taxonomical remarks on the ostracode genus Hollinella Coryell. Mededelingen Rijks Geologische Dienst, N. S., 21, 81-91.

Bless, M. J. M. and Jordan, H. 1971. Classification of Paleocopid ostracodes belonging to the families Ctenoloculinidae, Hollinidae and Hollinellidae. In: Paléoécologie des ostracodes (Ed. H. J. Oertli). Bulletin Centre de Recherches Pau-SNPA, 5 suppl., 869-890.

Brice, D. 1970. Etude paléontologique et stratigraphique du Dévonien de l'Afghanistan. Contribution a la connaissance des brachiopodes et des polypiers rugueux. Notes Mémoires Moyen-Orient, 11, 1-364.

Casier, J. G. 1977. Les ostracodes des schistes à aspect «Matagne» de la partie supérieure du Frasnien de l'affleurement protégé de Boussu-en-Fagne, Belgique. Bulletin de l'Institut Royal des Sciences naturelles de Belgique, Sciences de la Terre, 51, 1-33.

Casier, J. G. 1986. Ponderodictya belliloci nov. spec., nouvelle espèce d'ostracode (Metacopida) dans le Frasnien des Bassins de Namur et de Dinant (Belgique et Nord de la France). Bulletin de la Société belge de Géologie, 95, 301-305.

Casier, J.G. 1987. Étude biostratigraphique et paléoécologique des ostracodes du sommet du Givétien et de la base du Frasnien à Ave-et-Auffe (Bord sud du bassin de Dinant, Belgique). Bulletin de la Société belge de Géologie, 96, 23-34.

Casier, J. G. 1989. Paléoécologie des Ostracodes au niveau de la limite des étages Frasnien et Famennien, à Senzeilles. Bulletin de l'Institut Royal des Sciences naturelles de Belgique, 59, 79-93.

Coryell, H. N. 1928. Some new Pennsylvanian Ostracoda. Journal of Paleontology, 2, 377-381.

Coryell, H. N. and Malkin, D. S. 1936. Some Hamilton ostracodes from Arkona, Ontario. American Museum Novitates, 891, 1-20.

García-López, S., Sanz López, J. y Pardo Alonso, M. V. 1999. Conodontos (bioestratigrafía, biofacies y paleotemperaturas) de los sinclinales de Almadén y Guadalmez (Devónico-Carbonífero Inferior), Zona Centroibérica meridional, España. Revista Española de Paleontología, $\mathbf{n}^{\circ}$ extr. Homenaje al Prof. J. Truyols, 161-172.
García Sansegundo, J., Lorenzo Álvarez, S. y Ortega Gironés, E. 1987. Mapa y memoria explicativa de la Hoja Almadén (808) del Mapa geológico de España a escala 1:50.000. Instituto Geológico y Minero de España, 60 pp.

Gozalo, R. 1994. Geología y Paleontología (Ostrácodos) del Devónico Superior de Tabuenca (NE de la Cadena Ibérica Oriental). Memorias del Museo Paleontológico de la Universidad de Zaragoza, 6, 1-291.

Gozalo, R. y Sánchez de Posada, L. C. 1986. Polyzygia neodevonica aragonensis, nueva subespecie de Ostrácodos del Devónico de la Cordillera Ibérica. Revista Española de Micropaleontología, 18, 415-421.

Guernet, C. et Lethiers, F. 1989. Ostracodes et recherche des milieux anciens: possibilités et limites. Bulletin de la Société Géologique de France, ser. 8, 5, 577-588.

Gürich, G. 1896. Das Paleozoikum im Polnischen Mittelgebirge. Verhandlungen der Russischen Kaiserlichen Mineralogischen Gesellschaft zu S. Petersburg, ser. 2, 32, 1-539.

Hall, J. 1860. Descriptions of new species of fossils from the Hamilton group of western New York, with notices of others from the same horizon in Iowa and Indiana. New York State Cabinet of Natural History Annual Report, 13, 79-94.

Kesling, R. V. 1952. Ostracods of the Family Hollinidae from the Ferron Point formation of Michigan. Contributions from the Museum of Paleontology, University of Michigan, 10, 45-57.

Lethiers, F. 1974. Ostracodes du passage FrasnienFamennien de Senzeilles (Ardenne). Palaeontographica, Abt. A, 147, 39-69.

Lethiers, F. 1982. Les Ostracodes du Dévonien Supérieur (Nord de la France, Belgique, Ouest du Canada). Thèese de l'Universite des Sciences et Techniques de Lille, Num. d'ordre 560, 1-489.

Lethiers, F. 1984. Zonation du Dévonien supérieur par les Ostracodes (Ardenne et Boulonnais). Revue de Micropaléontologie, 27, 30-12.

Lethiers, F. et Racheboeuf, P. 1993. Les ostracodes de la Formation de Kersadiou (Givetien du Massif Armoricain): biostratigraphie et paléoécologie. Geobios, 26, 595-607.

Lethiers, F., Le Févre, J., Vannier, J. et Weyant, M. 1985. Paléozoïque. In: Atlas de ostracodes de France (Ed. H. J. Oertli). Bulletin Centre de Recherches ExplorationProduction Elf-Aquitaine, Mémoires, 9, 33-88.

Loranger, D. M. 1963. Devonian microfauna from northeastern Alberta. Part 2 Ostracoda. Order Podocopida. Published by the author, Calgary, $53 \mathrm{pp}$.

Matern, H. 1929. Die Ostracoden des Oberdevons. I. Teil. Aparchitidae, Primitiidae, Zygobolbidae, Beyrichiidae, Kloedenellidae, Entomidae. Abhandlungen der Preußischen Geologische Landesanstalt, N.F., 118, 1-99.

Michel, M. P. 1972. Polyzygia Gürich (Ostracoda) in the Devonian of Asturias and Leon (Spain). Leidse Geologische Mededelingen, 48, 207-273.

Müller, G. 1968. Bohr. Röhren von unbenkannten Anneliden und anderen Organismen in Unterdevonischen Brachiopodenklappen aus der Eifel und dem Siegerland (Rheinisches Schiefergebirge). Inaugural Dissertation, Köln Universität, 121 pp. (inédito). 
Murchison, R. I. 1840. Description de quelques unes des coquilles fossiles les plus abondantes dans les couches devoniennes du Bas-Boulonnais. Bulletin Société Géologique de France, 11, 250-256.

Oertli, H. J. 1971. The aspect of ostracode faunas. A possible new tool in petroleum sedimentology. In: Paléoécologie des ostracodes (Ed. H. J. Oertli). Bulletin Centre de Recherches Pau-SNPA, 5 suppl., 137-151.

Olivé Davó, A., Portero García, J. M., Álvaro López, A., Pieren Pidal, A., Aguilar, M. J., Dabrio, C. y Nozal, F. 1989. Memoria explicativa de la Hoja Herrera del Duque (756) del Mapa geológico de España a escala 1:50.000. Instituto Tecnológico y GeoMinero de España, $92 \mathrm{pp}$.

Paeckelmann, W. 1942. Beitrage zur kenntnis devonischer Spiriferen. Abhandlungen des Reichsamts für bodenforschung, N.F., 197, 1-188.

Pardo, M. V. y García-Alcalde, J. L. 1984. Biostratigrafía del Devónico de la región de Almadén (Ciudad Real, España). Trabajos de Geología, Universidad de Oviedo, 14, 79-120.

Pardo Alonso, M. V. 1997. El Devónico meridional de la Zona Centroibérica. Tesis Doctoral, Universidad de Oviedo, 472 pp. (inédito)

Pardo Alonso, M. V. 1999. Invertebrados marinos del Paleozoico Superior de Castilla - La Mancha. In: La huella del pasado: Fósiles de Castilla - La Mancha (Coords. E. Aguirre e I. Rábano). Patrimonio histórico. Arqueología - Castilla La Mancha, Toledo, 51-73.

Pardo Alonso, M. V. 2000. Presencia del género Eoparaphorhynchus en el Fameniense de la Zona Centroibérica suroriental (Ciudad Real, España). I Congreso Ibérico de Paleontología - XVI Jornadas de la Sociedad Española de Paleontología, libro de resúmenes, 264.

Pardo Alonso, M. V. y García-Alcalde, J. L. 1994. El Devónico de la Zona Centroibérica. Comunicaciones $X$ Jornadas Paleontología, Madrid, 153-156.

Pardo Alonso, M. V. y García-Alcalde, J. L. 1996. El Devónico de la Zona Centroibérica. Revista Española de Paleontología, $\mathbf{n}^{\circ}$ extraordinario, 72-81.

Pardo Alonso, M.V., López Buendía, A.M., Serrano, J. y Bastida, J. 1995. Presencia de pirofilita en reemplazamientos de conchas de espiriféridos (Brachiopoda) del Devónico de la Zona Centroibérica. (Macizo Hespérico, España). Cuadernos do Laboratorio Xeolóxico de Laxe, 20, 117-122.

Pokorný, V. 1951. The Ostracods of the Middle Devonian Red Coral Limestone of Celechovice. Sborník státního geologického Ústavu Ceskoslovenské, 17, 580-630.

Pokorný, V. 1965. Some palaeoecological problems in marine ostracod fauna, demonstrated on the Upper Cretaceous ostracodes of Bohemia, Czechoslovakia. Publicaciones della stazione Zoologica de Naples, 33 suppl., 462-479.

Puschmann, H. 1970. Das Paläozoikum der Nördlichen Sierra Morena am Beispiel der Mulde von Herrera del Duque (Spanien). Geologie, 19, 309-329.

Racheboeuf, P. R. 1981. Chonétacés (Brachiopodes) Siluriens et Dévoniens du Sud-Ouest de l'Europe
(Systématique-Phylogénie-BiostratigraphiePaléobiogéographie). Mémoires de la Société géologique et mineralogique de Bretagne, 27, 294 pp.

Racheboeuf, P. R. 1990. Les brachiopodes Chonetacés dans les assemblages benthiques siluriens et dévoniens. Palaeogeography, Palaeoclimatology, Palaeoecology, 81, 141-171.

Racheboeuf, P. R., Lethiers, F., Babin, C., Rolfe, W. D. I. et Marez, E. de. 1986. Les faunes du Dévonien supérieur d'Alange (province de Badajoz, Sud-Ouest de l'Espagne). Géologie Méditerranéenne, 12-13, 37-47.

Sánchez de Posada, L.C. y Bless, M. J. M. 1999. Hollinéllidos del Moscoviense Superior (Carbonífero) del este de Asturias (N de España). Revista Española de Paleontología, $\mathbf{n}^{\mathbf{0}}$ extr. Homenaje al Prof. J. Truyols, 29-41.

Soldevila Bartolí, J. 1992. La sucesión paleozoica en el sinforme de la Sierra de San Pedro (Provincias de Cáceres y Badajoz, SO de España). Estudios Geológicos, 48, 363-379.

Speyer, S.E. and Brett, C.E. 1988. Taphophacies models for epeiric sea environments: Middle Paleozoic examples. Palaeogeography, Palaeoclimatology, Palaeoecology, 63, 225-262.

Speyer, S.E. and Brett, C.E. 1991. Taphofacies controls. Background and episodic processes in fossil assemblage preservation. In: Taphonomy: Releasing the Data Locked in the Fossil Record (Eds. P. A. Allison and D. E. G. Briggs). Plenum Press, New York, 501-545.

Stewart, G. A. and Hendrix, W. E. 1945. Ostracoda of the Plum Brook Shale, Erie County, Ohio. Journal of Paleontology, 19, 87-95.

Thayer, C.W. 1975. Morphologic adaptations of benthic marine invertebrates to soft substrates. Journal of Marine Research, 33, 177-189.

Vergés, J. 1983. Estudio del Complejo vulcano-sedimentario del Devónico y de la estructura de la terminación oriental del sinclinal de Almadén (Ciudad Real). In: Libro Jubilar J. M. Ríos (Coord. J. A. Comba). Instituto Geológico y Minero de España, Madrid, 3, 215-229.

Verneuil, E. de (in Prado, C. de et Verneuil, E. de) 1850. Note géologique dur les terrains de Sabero et de ses environs dans les montagnes de Leon (Espagne), suivie dúne description des fossiles de ces terrains. Bulletin Société Géologique de France, ser. 2, 7, 137-186.

Whatley, R.C. 1983. The application of Ostracoda to palaeoenvironmental analysis. In: Applications of Ostracoda (Ed. R.F. Maddocks). University of Houston, Houston, 51-77.

Whatley, R. C. 1988. Population structure of ostracods: some general principles for the recognition of palaeoenvironments. In: Ostracoda in the Earth Sciences (Eds. P. De Deckker, J.-P. Colin and J.-P. Peypouquet). Elsevier, Amsterdan-Oxford-New York-Tokyo, 245-256.

Ziegler, W. 1996. Conodonten-Zonen, globale; aktueller Stand; Stand:1985. In: Devon-Korrelationstabelle (Ed. K. Weddige). Senckenbergiana lethaea, 76, 282.

Manuscrito recibido: 23 de mayo, 2001 Manuscrito aceptado: 29 de octubre, 2001 